\title{
A classification of the ground states and topological defects in a rotating two-component Bose-Einstein condensate
}

\author{
Peter Mason ${ }^{1,2}$ and Amandine Aftalion ${ }^{3}$ \\ ${ }^{1}$ Laboratoire de Physique Statistique, École Normale Supérieure, UPMC Paris 06, \\ Université Paris Diderot, CNRS, 24 rue Lhomond, 75005 Paris, France \\ ${ }^{2}$ Institut Jean Le Rond D'Alembert, UMR 7190 CNRS-UPMC, 4 place Jussieu, 75005 Paris, France \\ ${ }^{3}$ CNRS \&G Université Versailles-Saint-Quentin-en-Yvelines, \\ Laboratoire de Mathématiques de Versailles, CNRS UMR 8100, \\ 45 avenue des États-Unis, 78035 Versailles Cédex, France
}

(Dated: June 6, 2018)

\begin{abstract}
We classify the ground states and topological defects of a rotating two-component condensate when varying several parameters: the intracomponent coupling strengths, the intercomponent coupling strength and the particle numbers. No restriction is placed on the masses or trapping frequencies of the individual components. We present numerical phase diagrams which show the boundaries between the regions of coexistence, spatial separation and symmetry breaking. Defects such as triangular coreless vortex lattices, square coreless vortex lattices and giant skyrmions are classified. Various aspects of the phase diagrams are analytically justified thanks to a non-linear $\sigma$-model that describes the condensate in terms of the total density and a pseudo-spin representation.
\end{abstract}

PACS numbers:

\section{Introduction}

Bose Einstein condensates (BECs) provide an excellent environment to study experimentally and theoretically a rich variety of macroscopic quantum phenomena. In a rotating single component condensate, topological defects to the order parameter often manifest themselves as vortices that correspond to a zero of the order parameter with a circulation of the phase. When they get numerous, these vortices arrange themselves on a triangular lattice. In fact, vortices were first observed in twocomponent BEC's [1]. Since then two-component BECs and the topological excitations within have been experimentally realised in a number of configurations: a single isotope that is in two different hyperfine spin states 1 [ 7], two different isotopes of the same atom [8] or isotopes of two different atoms [9 11].

When a two-component condensate is under rotation, topological defects of both order parameters are created which lead to more exotic defects such as singly or multiply quantised skyrmions [7, 12-16]. Analogy with the Skyrme model from particle physics is often invoked to represent the defects [17, 18]. The singly quantised skyrmions contain a vortex in one component which has the effect of creating a corresponding density peak in the other component. These singly quantised skyrmions are often referred to as coreless vortices. Once numerous, these vortices and peaks arrange themselves in (coreless vortex) lattices, that can be either triangular or square. Other defects such as vortex sheets or giant skyrmions can also be observed [19 21]. The aim of this paper is to classify the type of defects according to the different parameters of the problem restricting ourselves to twodimensions. While this paper will only concern itself with magnetically trapped two-component BEC's, there is ac- tive research into spinor condensates (for a recent review see [23]).

In the mean-field regime, the two-component BoseEinstein condensate at zero temperature is described in terms of two wave functions (order parameters), $\Psi_{1}$ and $\Psi_{2}$, respectively representing component- 1 and component-2. The two-component condensate is placed into rotation about the $z$-axis with $\bar{\Omega}=\bar{\Omega} \hat{z}$ where $\bar{\Omega}$ is the rotation frequency assumed to apply equally to both components. The Gross-Pitaevskii (GP) energy functional of the rotating two-component, two-dimensional $\mathrm{BEC}$ is then given by

$$
\begin{aligned}
& E\left[\Psi_{1}, \Psi_{2}\right]=\int \sum_{k=1,2}\left(\frac{\hbar^{2}}{2 m_{k}}\left|\nabla \Psi_{k}\right|^{2}+V_{k}(\boldsymbol{r})\left|\Psi_{k}\right|^{2}\right. \\
& \left.-\hbar \bar{\Omega} \Psi_{k}^{*} L_{z} \Psi_{k}+\frac{U_{k}}{2}\left|\Psi_{k}\right|^{4}\right)+U_{12}\left|\Psi_{1}\right|^{2}\left|\Psi_{2}\right|^{2} \quad d^{2} r
\end{aligned}
$$

where $r^{2}=x^{2}+y^{2}$, and $V_{k}(\boldsymbol{r})=m_{k} \omega_{k}^{2} r^{2} / 2$ are the harmonic trapping potentials with trapping frequencies $\omega_{k}$, centered at the origin. Here $m_{1}$ and $m_{2}$ are the masses of the bosons in component- 1 and component- 2 respectively, and $\hbar$ is Planck's constant. The angular momentum is in the $z$-axis and is defined as $L_{z}=i[\hat{\boldsymbol{z}} \cdot(\boldsymbol{r} \times \boldsymbol{p})]$ for linear momentum $\boldsymbol{p}$. The energy functional (1) contains three interaction constants: $U_{k}$ representing the internal interactions in component $k$, and $U_{12}$ representing the interactions between the two components.

The time-dependent coupled Gross-Pitaevskii (GP) equations are obtained from a variational procedure, $i \hbar \partial \Psi_{k} / \partial t=\delta E / \delta \Psi_{k}^{*}$, on the energy functional (11). Let $\tilde{\omega}=\left(\omega_{1}+\omega_{2}\right) / 2$ be the average of the trapping frequencies of the two components and introduce the reduced mass $m_{12}$ such that $m_{12}^{-1}=m_{1}^{-1}+m_{2}^{-1}$. 
The GP energy and the coupled GP equations can be non-dimensionalised by choosing $\tilde{\omega}^{-1}$, $\hbar \tilde{\omega}$ and $r_{0}=$ $\sqrt{\hbar /\left(2 m_{12} \tilde{\omega}\right)}$ as units of time, energy and length respectively. On defining the non-dimensional intracomponent coupling parameters $g_{k}=2 U_{k} m_{12} / \hbar^{2}$ and the intercomponent coupling parameter $g_{12}=2 U_{12} m_{12} / \hbar^{2}\left(\equiv g_{21}\right)$, the dimensionless coupled GP equations read

$$
\begin{aligned}
i \frac{\partial \psi_{k}}{\partial t}=- & \frac{m_{12}}{m_{k}}\left(\nabla-i \boldsymbol{A}_{k}\right)^{2} \psi_{k}+\frac{m_{k}}{4 m_{12}}\left(\frac{\omega_{k}^{2}}{\tilde{\omega}^{2}}-\Omega^{2}\right) r^{2} \psi_{k} \\
& +g_{k}\left|\psi_{k}\right|^{2} \psi_{k}+g_{12}\left|\psi_{3-k}\right|^{2} \psi_{k},
\end{aligned}
$$

for $\Omega=\bar{\Omega} / \tilde{\omega}$ and where

$$
\boldsymbol{A}_{k}=\frac{1}{2} \frac{m_{k}}{m_{12}} \boldsymbol{\Omega} \times \boldsymbol{r},
$$

for $\boldsymbol{\Omega}=(0,0, \Omega)$ and $\boldsymbol{r}=(x, y, 0)$. The ground state of the energy (11) or the GP equations (2) is determined by preserving the normalisation condition which in this paper is either taken to be

$$
\int\left|\psi_{k}\right|^{2} \quad d^{2} r=N_{k}
$$

where $N_{k}$ is the total particle number of the $k$ thcomponent, or

$$
\int\left(\left|\psi_{1}\right|^{2}+\left|\psi_{2}\right|^{2}\right) \quad d^{2} r=N_{1}+N_{2} .
$$

The following sections will only consider repulsive interactions so that $g_{1}, g_{2}$ and $g_{12}$ are always non-negative. In order to separate the regimes of interest, a nondimensional parameter combining these $g_{k}$ and $g_{12}$ will appear:

$$
\Gamma_{12}=1-\frac{g_{12}^{2}}{g_{1} g_{2}}
$$

Furthermore to simplify matters, we introduce ratio parameters $\eta$ and $\xi$ such that

$$
m_{1}=\eta m_{2} \text { and } \omega_{1}=\xi \omega_{2} \text { with }\{\eta, \xi\}>0 .
$$

The effective trapping potentials for each component coming from equation (2) are then, respectively,

$$
\begin{aligned}
& V_{1}^{\mathrm{eff}}(r)=(\eta+1)\left(\frac{\xi^{2}}{(\xi+1)^{2}}-\frac{1}{4} \Omega^{2}\right) r^{2}, \\
& V_{2}^{\mathrm{eff}}(r)=\frac{(\eta+1)}{\eta}\left(\frac{1}{(\xi+1)^{2}}-\frac{1}{4} \Omega^{2}\right) r^{2} .
\end{aligned}
$$

It follows that the limiting rotational frequency for each component is $\Omega_{1}^{\lim }=2 \xi /(\xi+1)$ and $\Omega_{2}^{\lim }=2 /(\xi+1)$, so that it is necessary to consider a rotational frequency such that $0<\Omega<\Omega^{\lim }=\min \left\{\Omega_{1}^{\lim }, \Omega_{2}^{\lim }\right\}$.
Experimentally, it is often the case that the $U_{k}, m_{k}, \omega_{k}$ and $N_{k}$ are of the same order, so that much of the theoretical and numerical analysis concerning two-component condensates has assumed equality of these parameters. In the case where the intracomponent coupling strengths are equal, Kasamatsu et al. [19, 20] produced a numerical phase diagram in terms of the rotation and the intercomponent coupling strengths: they found phase separation regions with either vortex sheets or droplet behavior and regions of coexistence of the components with coreless vortices, arranged in triangular or square lattices.

In this paper, we present a classification of the ground states and various types of topological defect when the intracomponent coupling strengths are distinct. Depending on the magnitude of the various parameters the components can either coexist, spatially separate or exhibit symmetry breaking. A richer phenomenology of topological defects is then found.

Much of the analysis carried out to investigate the ground states and topological defects will use a a nonlinear sigma model. It has been introduced previously in the literature [19, 24] for $\eta=\xi=1$ but we will generalise this to the cases $\eta$ and $\xi$ different from 1 . This involves writing the total density as

$$
\rho_{T}=\left|\psi_{1}\right|^{2}+\eta\left|\psi_{2}\right|^{2} .
$$

A normalised complex-valued spinor $\chi=\left[\chi_{1}, \chi_{2}\right]^{T}$ is introduced from which the wave functions are decomposed as $\psi_{1}=\sqrt{\rho_{T}} \chi_{1}, \psi_{2}=\sqrt{\rho_{T} / \eta} \chi_{2}$ where $\left|\chi_{1}\right|^{2}+\left|\chi_{2}\right|^{2}=1$. Then we define the spin density $\boldsymbol{S}=\bar{\chi} \boldsymbol{\sigma} \chi$ where $\boldsymbol{\sigma}$ are the Pauli matrices. This gives the components of $\boldsymbol{S}$ as

$$
\begin{aligned}
& S_{x}=\chi_{1}^{*} \chi_{2}+\chi_{2}^{*} \chi_{1}, \\
& S_{y}=-i\left(\chi_{1}^{*} \chi_{2}-\chi_{2}^{*} \chi_{1}\right), \\
& S_{z}=\left|\chi_{1}\right|^{2}-\left|\chi_{2}\right|^{2},
\end{aligned}
$$

with $|\boldsymbol{S}|^{2}=1$ everywhere. We will write the GP energy (11) in terms of $\rho_{T}$ and $\boldsymbol{S}$ that will allow us to derive information on the ground state of the system.

The paper is organised as follows. The different regions of the $\Omega-\Gamma_{12}$ phase diagrams are outlined in Sect. II with a detailed analysis of the range of ground states and topological defects. Then the non-linear $\sigma$-model is developed in Sect. III to analyse the ground states in terms of the total density. Lastly Sect. IV takes the second normalisation condition (5) and presents an example $\Omega-\Gamma_{12}$ phase diagram.

\section{The $\Gamma_{12}-\Omega$ Phase Diagram}

\section{A. Numerical Parameter Sets}

In this paper, three different configurations are considered, two of which relate directly to experimental config- 
urations. Firstly, we analyze a ${ }^{87} \mathrm{Rb}-{ }^{87} \mathrm{Rb}$ mixture with one isotope in spin state $\left|F=2, m_{f}=1\right\rangle$ and the other in state $|1,-1\rangle$. The complex relative motions between these two isotope components of rubidium were considered experimentally by Hall et al [2]. Here, the masses are equal $(\eta=1)$ and the transverse trapping potentials are equal, $\omega_{1}=\omega_{2}(\xi=1)$. The scattering lengths for each component are $a_{1}=53.35 \AA$ and $a_{2}=56.65 \AA$. The intracomponent coupling strengths $U_{k}$ for a twodimensional model are defined as $U_{k}=\sqrt{8 \pi} \hbar^{2} a_{k} /\left[m_{k} a_{z k}\right]$ $(k=1,2)$, and the intercomponent coupling strength is defined as $U_{12}=\sqrt{2 \pi} \hbar^{2} a_{12} /\left[m_{12} \tilde{a}_{z}\right]$, where $m_{12}$ is the reduced mass, given by $m_{12}^{-1}=m_{1}^{-1}+m_{2}^{-1}, a_{k}, a_{12}$ are the s-wave (radial) scattering lengths, $a_{z k}$ is the characteristic size of the condensate in the $z$ direction, and $\tilde{a}_{z}=\left(a_{z 1}+a_{z 2}\right) / 2$. In the experiment [2], the computations of approximate values of $a_{z 1}$ and $a_{z 2}$ can be made using a Thomas-Fermi approximation in the $z$ direction as explained in [25], chapter 17. This relies on the assumption that $N_{k} a_{k} / a_{z k}$ are large. Then, it is reasonable to describe the experiments through a two-dimensional model. For our simulations, we choose $g_{1}=0.0078$ and $g_{2}=0.0083$, which are consistent with the experimental data and we set $N_{1}=N_{2}=10^{5}$. We denote this parameter set as 'Experimental Set 1' (ES1).

Secondly, we tackle a ${ }^{41} \mathrm{~K}-{ }^{87} \mathrm{Rb}$ mixture with both isotopes in spin state $|2,2\rangle$, as was considered by Modugno et al 9]. Component-1 is identified with the ${ }^{41} \mathrm{~K}$ isotope and component- 2 with the ${ }^{87} \mathrm{Rb}$ isotope. In the experiment, the masses are different $(\eta \sim 0.48)$, but since $\eta \xi^{2}=1$, then $m_{1} \omega_{1}^{2}=m_{2} \omega_{2}^{2}$ so that both components experience the same trapping potential. Here the scattering lengths for each component are $a_{1}=31.75 \AA$ and $a_{2}=52.39 \AA$ and we choose intracomponent coupling strengths as $g_{1}=0.0067$ and $g_{2}=0.0063$. This set is denoted ES2.

Lastly, in order to make an analogy with previous theoretical studies, we consider the set (ES3) which contains a mixture chosen such that all the parameter groups are equal, i.e. here $g_{1}=g_{2}=0.0078$ and $N_{1}=N_{2}=10^{5}$ with equality of the $m_{k}$ and $\omega_{k}(\eta=\xi=1)$.

In each case, the features of the ground states will be explained as $g_{12}$ and $\Omega$ are varied. While the value of the $g_{k}$ are nominally fixed, the value of the intercomponent coupling strength, $g_{12}$, can be altered by Feshbach resonance (see for instance [26 29]), which allows for an extensive experimental range in $\Gamma_{12}$, given by (6) (keeping $\left.\Gamma_{12} \leq 1\right)$. Simulations are thus performed on the coupled GP equations (2) in imaginary time. In general, it is difficult to find the true minimizing energy state. But the use of various initial data converging to the same final state allows us to state that the true ground state will be of the same pattern as the one we exhibit.

We would like to note that while we have chosen these particular parameters, we have conducted extensive numerical simulations over a range of different parameters sets and find the sets presented here illustrate well the possible ground states.

\section{B. Classification of the Regions of the Phase Diagram}

The ground states can be classified according to

1. the symmetry properties

2. the properties of coexistence of the condensates or spatial separation.

When there is no rotation $(\Omega=0)$, as illustrated in Fig. 1] the geometry of the ground state can either be

- two disks (coexistence of the components and symmetry preserving state)

- a disk and an annulus (symmetry preserving with spatial separation of the components), which depends strongly on the fact that the $g_{k}, m_{k}, \omega_{k}$ are not equal

- droplets (symmetry breaking and spatial separation).

Under rotation, the different ground states can be classified according to the parameters $\Gamma_{12}$ and $\Omega$. When the condensates are two disks, or a disk and annulus, defects may break some symmetry of the system as $\Omega$ is increased. But the wave function retains some non trivial rotational symmetry. We will not refer to this as symmetry breaking since the bulk condensate keeps some symmetry. We will use the terminology symmetry breaking when the bulk completely breaks the symmetry of the system and is a droplet or has vortex sheets. We find that there are four distinct regions, determined by the geometry of the ground state.

Region 1: Both components are disk shaped, with no spatial separation. Above some critical velocity $\Omega$, coreless vortices appear: a vortex in one component which has the effect of creating a corresponding density peak in the other component. They arrange themselves either on a triangular or on a square lattice. Figure 2 provides a typical example of the density profiles.

Region 2: Vortex sheets. Under the effect of strong rotation, the components spatially separate and completely break the symmetry of the system. Nevertheless, they are approximately disk shaped with similar radii. Many vortices are nucleated that arrange themselves into rows that can have various patterns: either they can be striped or bent and are often disconnected from each other. A vortex sheet in one component corresponds to a region of macroscopic density in the other component. These features can be seen in the density plots of Fig. 31(c). 

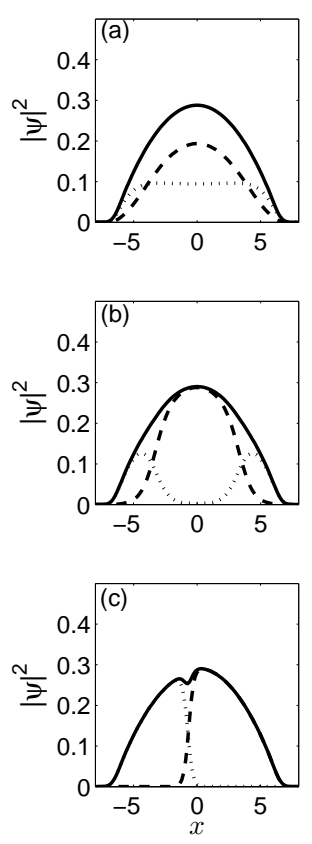

FIG. 1: Density (divided by $10^{4}$ ) plots along $y=0$ for component-1 (dashed lines), component-2 (dotted lines) and the total density (solid lines) in which the components (a) coexist both being disks $\left(\Gamma_{12}=0.1\right)$, (b) spatially separate to be a disk and an annulus $\left(\Gamma_{12}=0\right)$ and (c) have symmetry broken to be two droplets $\left(\Gamma_{12}=-0.3\right)$. The parameters are $g_{1}=0.0078, g_{2}=0.0083$ and $N_{1}=N_{2}=10^{5}$ with $\eta=\xi=1$ (set ES1). The angular velocity of rotation is $\Omega=0$. Distance is measured in units of $r_{0}$ and density in units of $r_{0}^{-2}$.

Region 3: Spatial separation preserving some symmetry. Here one component is a disk while the other component is an annulus and the disk fits within the annulus with a boundary layer region in which both components have microscopically small density as illustrated in Figure3(b) and 4(b). Under rotation, the topological defects can either be coreless vortex lattices in the disk and (but not always) corresponding isolated density peaks in the annulus, and/or a giant skyrmion at the boundary interface between the two components, as will be described later.

Region 4: Rotating droplets. The components spatially separate and have broken symmetry such that the centres of each condensate are different and each component contains a single patch of density as illustrated in Figures 3(a) and 4(a). In the rotating droplets, vortices can appear provided the rotation is greater than some critical value. These features can be seen in the density plots of Fig. 5 .

The $\Omega-\Gamma_{12}$ phase diagrams corresponding to the experimental parameter sets introduced above (sets ES1,
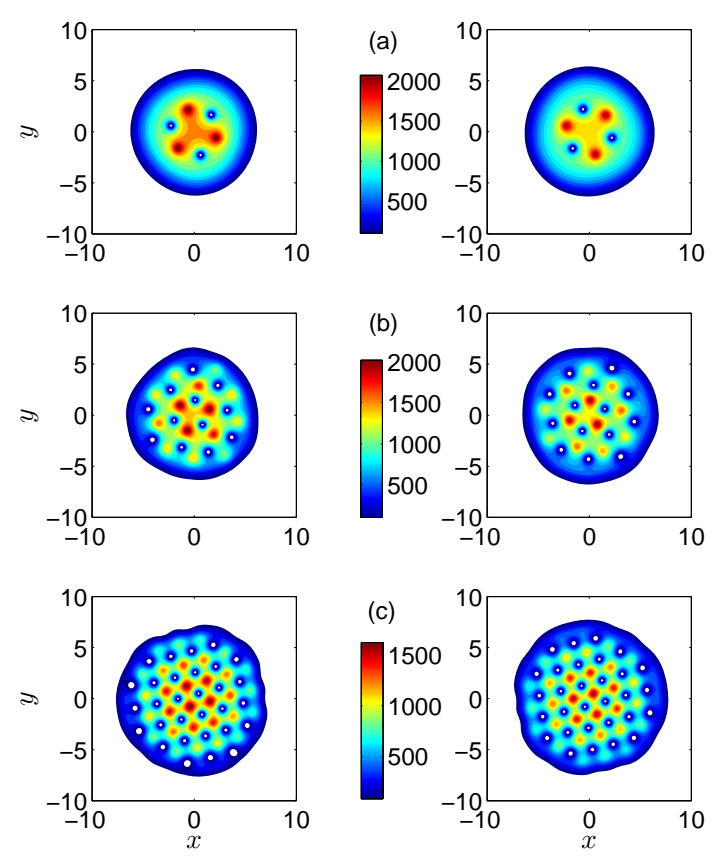

FIG. 2: (Color online) A series of density plots for component1 (left column) and component-2 (right column) in which the components coexist and are both disks. The parameters are $g_{1}=0.0078, g_{2}=0.0083$ and $N_{1}=N_{2}=10^{5}$ and with $\eta=\xi=1$ (set ES1) and $\Gamma_{12}=0.5$ (which gives $g_{12}=0.0057$ ). The angular velocity of rotation is $\Omega$ and it takes the values (a) 0.25 , (b) 0.5, and (c) 0.75. Vortices in one component have a corresponding density peak in the other component (coreless vortices). In (c) the coreless vortex lattice is square. At these parameters the components are in region 1 of the phase diagram of Fig. 6 Distance is measured in units of $r_{0}$ and density in units of $r_{0}^{-2}$.

ES2 and ES3) are shown in Fig.'s 6, 7] and 8. The last one is of similar type as the one reported by Kasamatsu et al [20] (there $g_{1}=g_{2}=4000$ and $N_{1}=N_{2}=1 / 2$ ).

New features can be observed in Fig 6 and 7 such as isolated density peaks that eventually vanish as $\Gamma_{12}$ is made more negative and the multiply quantised skyrmions at the interface between the disk component and the annular component in region 3. When $\{\eta, \xi\} \neq 1$ (Fig. 7), no region 2 is found to exist. This absence is easily explained by two factors: the onset of region 3 for (large) positive values of $\Gamma_{12}$ and the lack of vortices nucleated in component-1 (and to some extent in component-2).

We will now analyze in more detail some of the features of regions 1, 3 and 4 of the phase diagrams. Vortex sheets (region 2) have been analysed (mainly in the case of equal $g_{k}, m_{k}$ and $\left.\omega_{k}\right)$ in [30]. 

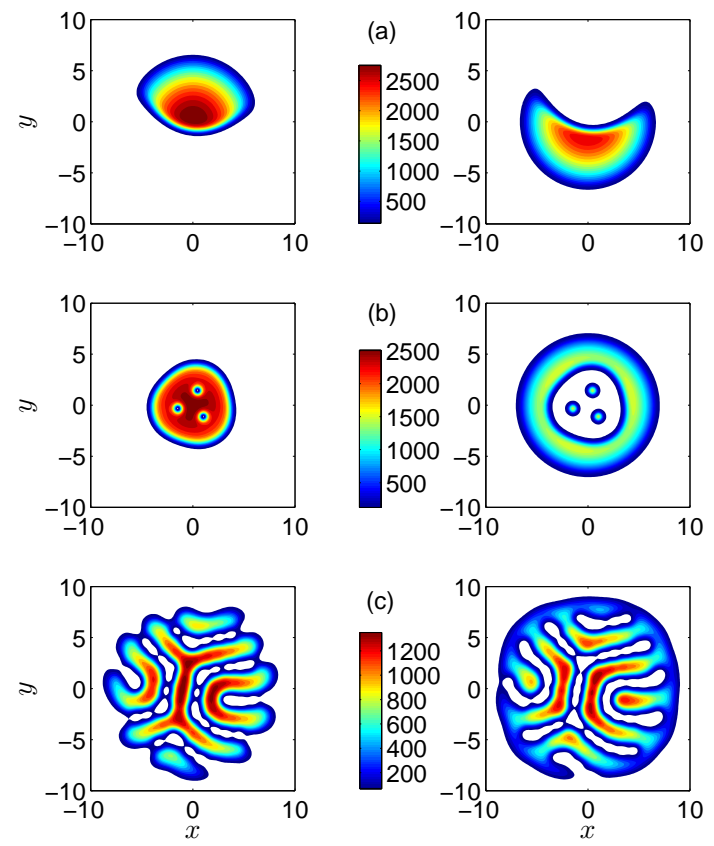

FIG. 3: (Color online) A series of density plots for component1 (left column) and component-2 (right column) in which the components have spatially separated. The parameters are $g_{1}=0.0078, g_{2}=0.0083$ and $N_{1}=N_{2}=10^{5}$ and with $\eta=\xi=1$ (set ES1) and $\Gamma_{12}=-0.3$ (which gives $g_{12}=$ $0.0092)$. The angular velocity of rotation is $\Omega$ and it takes the values (a) 0.1, (b) 0.5, and (c) 0.9. In (a) the components are rotating droplets (region 4 of Fig. 6), in (b) the components have spatially separated (but keep some symmetry) and have isolated density peaks (region 3 of Fig. 6) and in (c) there are vortex sheets present (region 2 of Fig. 6). Distance is measured in units of $r_{0}$ and density in units of $r_{0}^{-2}$.

\section{Analysis of the Features of the Phase Diagrams}

\section{Square lattices}

It is a specific property of two components to stabilize square lattices. A requirement for the existence of square lattices is the nucleation of many vortices in both components, something not permitted in ES2 (Fig. 7), where only a small number of vortices are ever nucleated in component 1.

Square lattices generally occur at high rotational velocities and examples have been observed experimentally [7] and numerically [19, 20, 31]. Mueller and Ho 32 and later Keçeli and Öktel 33] have analysed the transition from triangular to square lattices as $\Gamma_{12}$ is varied in two-component condensates when $g_{1} \sim g_{2}$ and $\Omega$ is such that the condensate is in the lowest Landau level (LLL). Providing $\Omega$ is large, according to 32, 33.
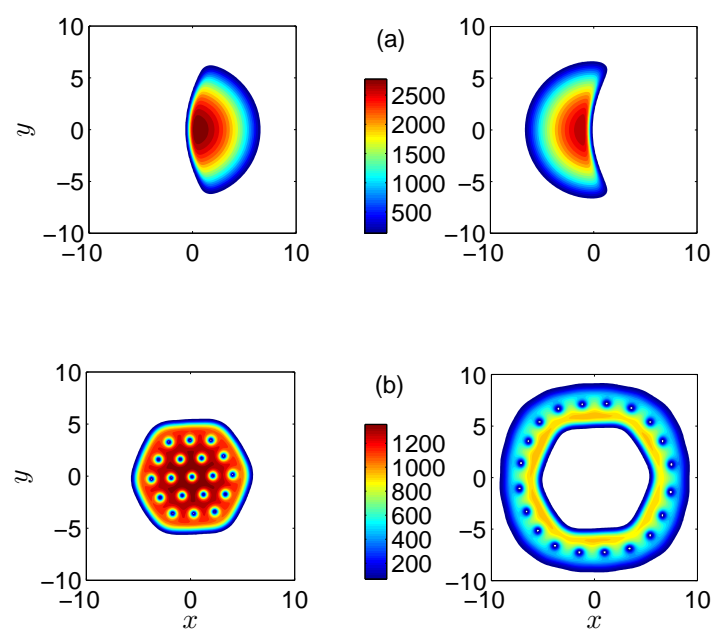

FIG. 4: (Color online) A series of density plots for component1 (left column) and component-2 (right column). The parameters are $g_{1}=0.0078, g_{2}=0.0083$ and $N_{1}=N_{2}=10^{5}$ and with $\eta=\xi=1$ (set ES1) and $\Gamma_{12}=-1.3$ (which gives $g_{12}=0.0122$ ). The angular velocity of rotation is $\Omega$ and it takes the values (a) 0 and (b) 0.9. In (a) the components are rotating droplets (region 4 of Fig. 6) while in (b) the components have spatially separated (but keep some symmetry) and there are no isolated density peaks (region 3 of Fig. 6). Distance is measured in units of $r_{0}$ and density in units of $r_{0}^{-2}$.

the value of $\alpha=\sqrt{1-\Gamma_{12}}$ determines whether the vortex lattices are triangular $(0<\alpha<0.172)$, in transition to becoming square $(0.172<\alpha<0.373)$ or square $(\alpha>0.373)$. Therefore, assuming that, in the example parameters of $g_{1}=0.0078, g_{2}=0.0083$ and $\eta=\xi=1$ (set ES1), the $g_{k}$ are sufficiently close enough, the square lattice should first appear at $\alpha=0.37$, or equivalently $\Gamma_{12}=0.86$. Comparing this to the numerically obtained values, where the square lattice first appears for $0.8<\Gamma_{12}<0.9$ in Fig. 6, the agreement seems good. It will be interesting to see how the analysis of [32, 33] has to be modified when $g_{1}$ becomes distant from $g_{2}$.

Nevertheless, the square lattices are present for lower rotational velocities at which the LLL is not necessarily valid. In a forthcoming paper, using the nonlinear sigma model presented below, we hope to derive a vortex energy in terms of the positions of vortices. The ground state of this energy indeed leads to square lattices in some range of parameters (see also Section III.E). It turns out that this vortex energy is similar to that of Barnett et al 34, 35. 

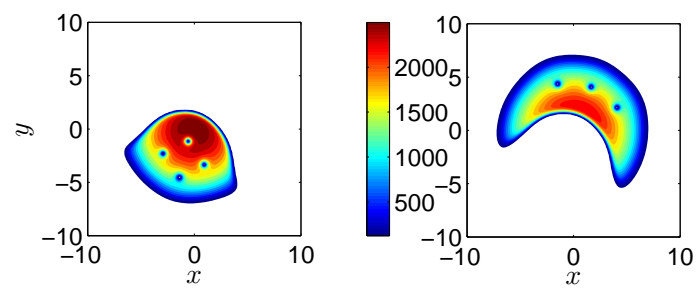

FIG. 5: (Color online) A density plot for component-1 (left column) and component-2 (right column) showing examples of vortex nucleation in rotating droplets. The parameters are $g_{1}=0.0078, g_{2}=0.0083$ and $N_{1}=N_{2}=10^{5}$ and with $\eta=\xi=1$ (set ES1) and $\Gamma_{12}=-9$ and $\Omega=0.5$. At these parameters the components are in region 4 of the phase diagram of Fig. 6. Distance is measured in units of $r_{0}$ and density in units of $r_{0}^{-2}$.

\section{Rotating droplets}

In the parameter range of region 4, symmetry breaking with spatial separation occurs. When the condensate is not under rotation, the ground states of the two components will be 'half ball'-like structures, as can be seen in Fig. 4(a) (if $g_{1}=g_{2}$ and $\eta=\xi=1$, then the two components are exactly half-balls). The difference between the intercomponent strengths introduces a curvature to the inner boundary of each component with the component that corresponds to larger $g_{k}$ having positive curvature and the other component having negative curvature. This curvature depends on both $\Gamma_{12}$ and $\Omega$. If $\Gamma_{12}$ is held constant, then the curvature increases as $\Omega$ increases. When $\Gamma_{12}$ goes to $-\infty$, the droplets approach half-balls.

Vortex nucleation is also seen in region 4; see Fig. 5 , In this figure there are four vortices in component-1 and three vortices in component-2. The number of vortices in each component will increase as $\Omega$ is increased but this increase will also increase the curvature of the inner boundaries of the components, thus preventing the vortices aligning themselves into vortex sheets. Examples of the rotating droplet ground states can be seen in Fig. 4(a), and further examples can be found in [22, 36 39].

\section{Spatial separation preserving some symmetry: disk plus annulus}

Region 3 is defined by the $\Gamma_{12}$ and $\Omega$ in which the condensates have spatially separated components but still possess some symmetry about the origin: the disk component- 1 sits securely within the annular component2 (see Fig.'s 3(b) and 4(b)). There is a boundary layer evidenced where the outer edge of the disk overlaps the
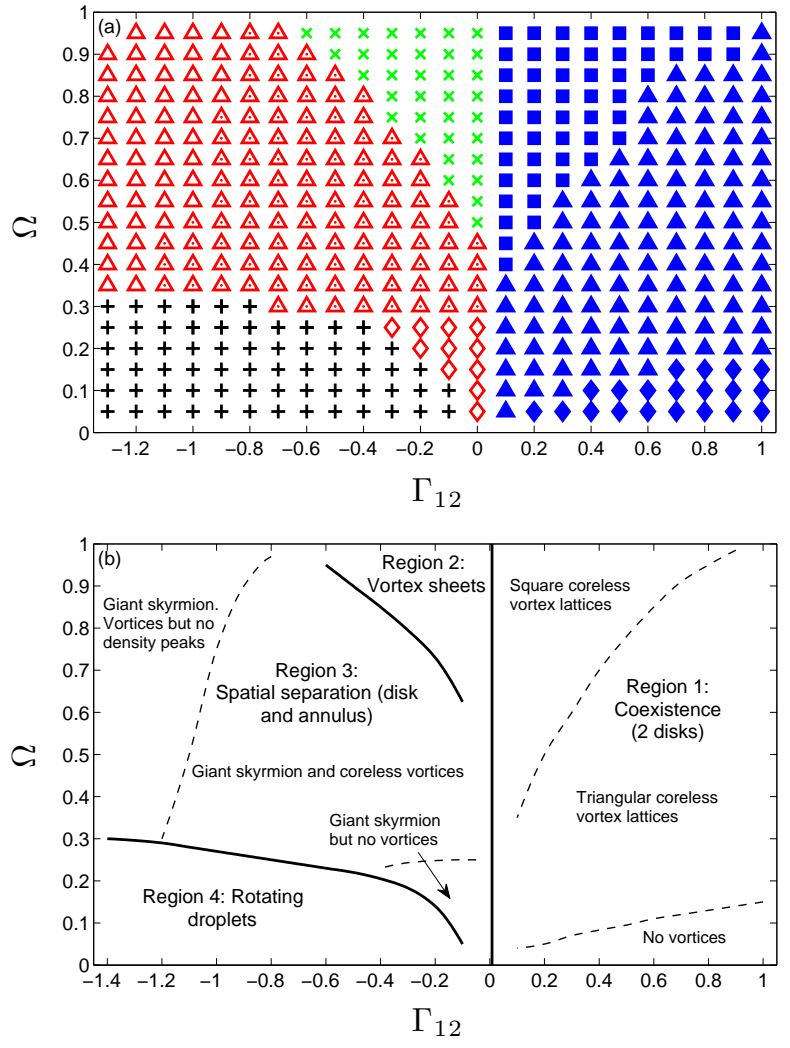

FIG. 6: (Color online) $\Omega-\Gamma_{12}$ phase diagrams for parameters $g_{1}=0.0078, g_{2}=0.0083, N_{1}=N_{2}=10^{5}$ with $\eta=\xi=1$ (set ES1) with normalisation taken over the individual components (Eq. (4)). (a) Numerical simulations where triangles (squares) indicate that the vortex lattice in both components is triangular (square) and diamonds that no vortices have been nucleated. Filled triangles, squares and diamonds are where the two components are disk-shaped and coexist. The empty triangles and empty diamonds are where the two components have spatially separated: one component is a disk and the other an annulus with a giant skyrmion at the boundary layer; those triangles with a dot in the centre represent the appearance of coreless vortices inside the annulus. The crosses ' $\mathrm{x}$ ' are where the two components have broken symmetry and are vortex sheets and the crosses ' + ' are rotating droplets. (b) A schematic representation of the numerical simulations. The solid lines indicate the boundary between different identified regions (determined by the geometry of the ground state) and the dashed lines the boundary between triangular and square lattices in region 1 and the boundary between peaks and no peaks in region 3 . The boundary between region 1 and the others can be calculated analytically by Eq. (39) to give $\Gamma_{12}=0.008$. The unit of rotation is $\tilde{\omega}$.

inner edge of the annulus.

Let us describe the onset of region 3 from region 4 , captured in Fig. 3 (sub-image (a) to (b) or (c) to (b)). For a particular $\Gamma_{12}$, as $\Omega$ is increased, then the curvature increases to such an extent that the components develop constant non-zero curvature, and are identified as a disk 

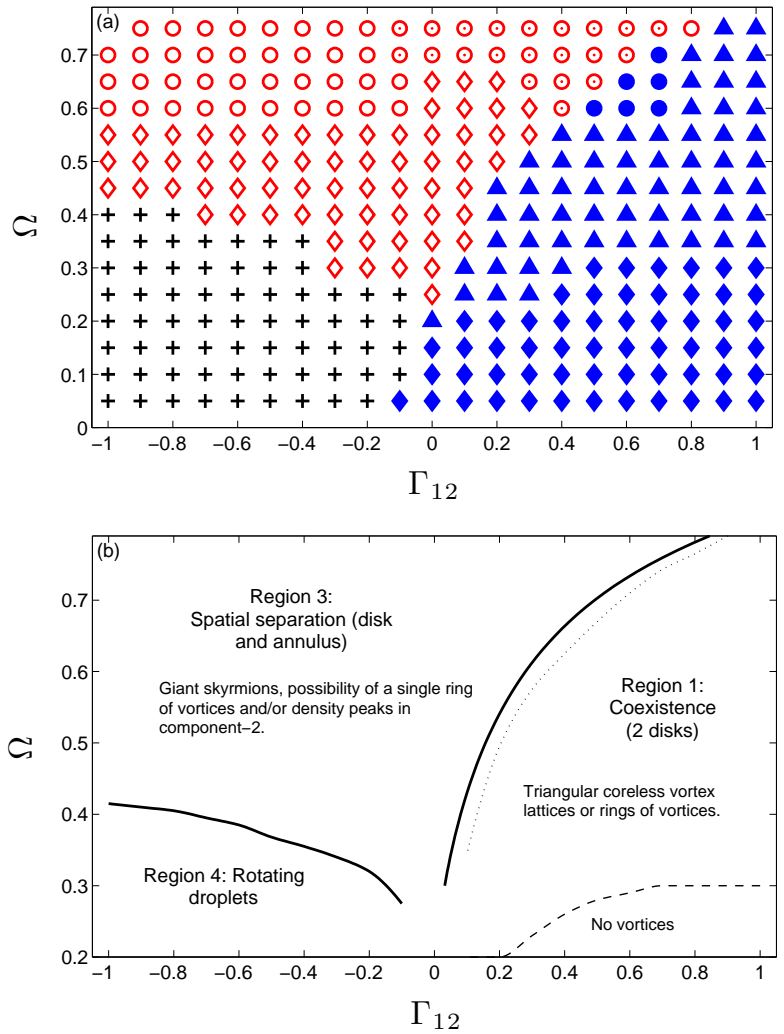

FIG. 7: (Color online) $\Omega-\Gamma_{12}$ phase diagrams for parameters $g_{1}=0.0067, g_{2}=0.0063, N_{1}=N_{2}=10^{4}$ with $\eta=0.48$ and $\eta \xi^{2}=1$ (set ES2) with normalisation taken over the individual components (Eq. (4)). (a) Numerical simulations where triangles indicate that the vortex lattice in component- 2 is triangular, circles that component- 2 contains rings of vortices and diamonds where no vortices have been nucleated. Filled triangles, circles and diamonds are where the two components are disk-shaped and coexist. The empty circles and empty diamonds are where the two components have spatially separated: one component is a disk and the other an annulus with a giant skyrmion at the boundary layer; those circles with a dot in the centre represent the appearance of coreless vortices inside the annulus. The crosses ' + ' are where the two components have broken symmetry and are rotating droplets. (b) A schematic representation of the numerical simulations. The solid lines indicate the boundary between different identified regions (determined by the geometry of the ground state). The boundary between region 1 and region 3 is also calculated analytically by Eq. (39) (dotted line). For these parameters there is no region 2. The unit of rotation is $\tilde{\omega}$.

and an annulus. Conversely, if $\Omega$ is held constant and $\Gamma_{12}$ is pushed to more negative values, then the curvature decreases.

Under rotation, defects can be observed: coreless vortices and giant skyrmions. Coreless vortices sit inside the disk while giant skyrmions are observed in the boundary layer.

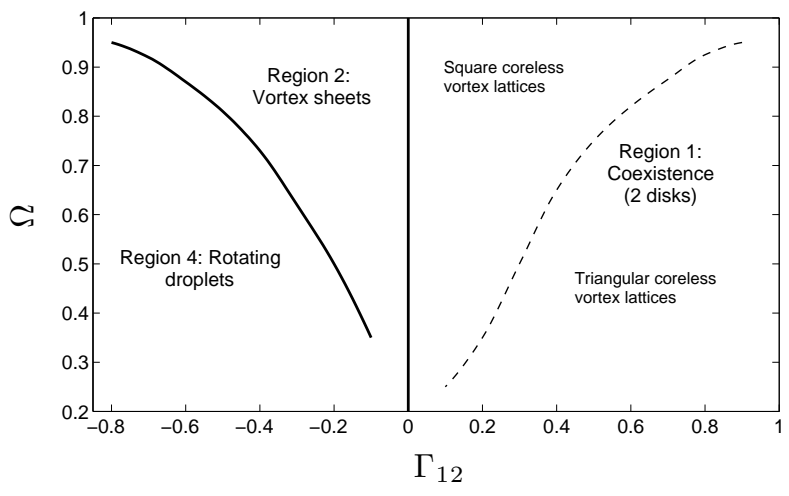

FIG. 8: A schematic $\Omega-\Gamma_{12}$ phase diagram for $g_{1}=g_{2} \equiv g=$ 0.0078, $N_{1}=N_{2}=10^{5}$ where $\eta=\xi=1$ (set ES3) and with the normalisation taken over the individual components (Eq. (41)). The solid lines indicate the boundary between different identified regions (determined by the geometry of the ground state) and the dashed lines the boundary between triangular and square lattices. For these parameters there is no region 3. The unit of rotation is $\tilde{\omega}$.

\section{Coreless Vortices in the disk plus annulus case}

In the geometry of disk plus annulus, the vortices in the inside disk have corresponding isolated density peaks inside the annulus, hence in the microscopic density regions, as illustrated in Fig. 3(b). Thus any vortex lattice structure produced in component- 1 is replicated by a peak lattice structure in component-2, i.e. there is the continuing presence of coreless vortex lattices in the spatially separated condensates [40].

As the number of vortices in the disk increases with increasing angular velocity, the number of density peaks inside the central hole of the annulus likewise increases. This has the effect that the central hole of the annulus can be masked at high angular velocities by the multiple occurrence of the density peaks. A recent analytical understanding of the interaction between vortices and peaks has been provided by [41]. It may be extended to the case of the disk and annulus, for which the average density in the central hole is very small for one component.

Pushing $\Gamma_{12}$ to lower values has the effect of reducing the size of the boundary layer between the disk and annular components, but also the isolated density peaks disappear; see Fig. 4(b). A recent work has analysed, from an energy perspective, the preference for the ground state to contain or not contain density peaks [42].

Figure 9 plots the maximum peak density of the density peaks in component-2 as a function of $\Gamma_{12}$ for the parameters of Fig. 6 and with $\Omega=0.5$ and $\Omega=0.75$. The disjoint region when $\Omega=0.75$ for $-0.4<\Gamma_{12}<0.1$ on Fig. 9 is due to the absence of density peaks in component-2 as a result of the appearance of vortex 


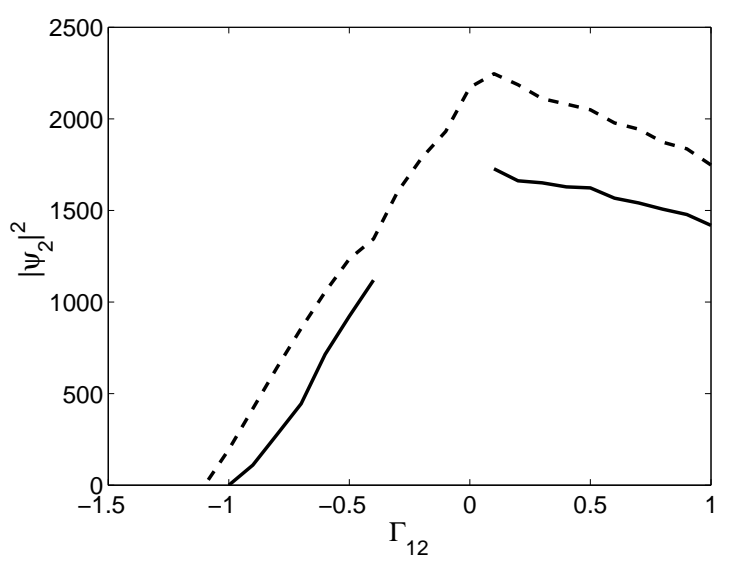

FIG. 9: Plots of maximum peak density in component-2 against $\Gamma_{12}$ for $\Omega=0.5$ (dashed line) and $\Omega=0.75$ (solid line) when $g_{1}=0.0078, g_{2}=0.0083, N_{1}=N_{2}=10^{5}$ and with $\eta=\xi=1$ (set ES1). The disjoint region when $\Omega=0.75$ for $-0.4<\Gamma_{12}<0.1$ is due to the absence of isolated density peaks in component-2 as a result of the appearance of vortex sheets. Density is measured in units of $r_{0}^{-2}$.

sheets (region 4). We see from Fig. 9, that the maximum peak density occurs when $\Gamma_{12} \sim 0.01$ which is the value at which the components begin to spatially separate (the transition between region 1 and region 3 ) in set ES1. For $\Gamma_{12}$ and $\Omega$ that take values outside of region 1 , the maximum density of the peaks decreases linearly as $\Gamma_{12}$ increases. The maximum density approaches microscopically small values for $\Gamma_{12} \sim-1.1$ when $\Omega=0.5$ and for $\Gamma_{12} \sim-1$ when $\Omega=0.75$. An example of the transition can be seen in Fig.'s 3 (b) and 4 (b).

\section{Giant skyrmion}

A boundary layer between the overlap of component-1 and component-2 is present for all values of $\Gamma_{12}$ in region 3 but reduces in width as $\Gamma_{12}$ becomes more negative (indeed the boundary layer disappears only as $\Gamma_{12} \rightarrow-\infty$ ). There are additional topological defects that occur in the boundary layer that are not discernible on a traditional density plot. These topological defects' presence can be observed in a phase profile, however a better visualisation is to use the pseudospin representation (9)-(10). One can plot the functions $S_{x}, S_{y}$ and/or $S_{z}$ which reveal the presence of all the topological defects - the coreless vortices (singly quantised skyrmions) that have already been visualised on the plots, and the new defect, a multiply quantised skyrmion.

The distinct nature of the two types of topological defect can be clearly seen in Fig. 10(a,b) where an $S_{x}$ plot and an $\left(S_{x}, S_{y}\right)$ vectorial plot are shown respectively. A density plot of each component for these same parame-
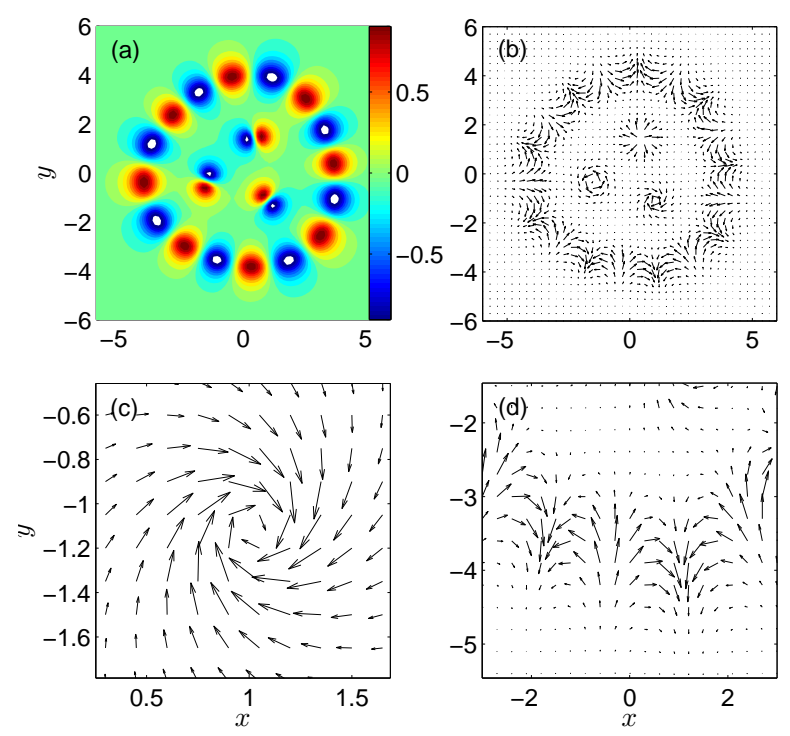

FIG. 10: (Color online) Plots of (a) $S_{x}$ and (b) a vectorial plot of $\left(S_{x}, S_{y}\right)$ for $\Gamma_{12}=-0.3$ and $\Omega=0.5$ when $g_{1}=0.0078$, $g_{2}=0.0083, N_{1}=N_{2}=10^{5}$ and with $\eta=\xi=1$ (set ES1). The ring of skyrmions, at $r=r_{s}$, traces the boundary layer and the coreless vortices exist for $r<r_{s}$. Blow-ups (c) and (d) of (b) highlight the nature of the two types of topological defect; (c) a coreless vortex at $(1,-1)$ and (d) a section of the multiply quantised skyrmion. The respective density plots for this case are shown in Fig. 3(b). Distance is measured in units of $r_{0}$.

ters is seen in Fig. 3(b). The coreless vortices evident in Fig. 3(b) are again clearly evident in Fig. 10(a) and (b). A blow-up of the region close to one of (the three) coreless vortices in the $\left(S_{x}, S_{y}\right)$ vectorial plot, centred at $(1,-1)$ and exhibiting circular disgyration is shown in Fig. 10(c). The texture of $\boldsymbol{S}$ can also exhibit crossand radial- disgyration [43]. Conversely, the multiply quantised skyrmion, not present in the density plots of Fig. 3(b), can clearly be seen in both Fig. 10(a) and (b). The multiply quantised skyrmion forms a ring along the boundary layer. A blow-up around $(0,-3.5)$ for the $\left(S_{x}, S_{y}\right)$ vectorial plot again shows the multiply quantised nature of this defect. A multiply quantised skyrmion was evidenced by [21] who termed it a giant skyrmion, a terminology retained in this paper. Increasing the rotation results in an increase in both the number of coreless vortices and in the multiplicity of the giant skyrmion. Ref 21] gave a relationship, $m=n+1$, between the multiplicity of the giant skyrmion $n$ and the total circulation $m$ in the central hole of component-2. However the numerical simulations here suggest that a more appropriate correlation is provided by $m=n+l$ where $l$ is the number of isolated coreless vortices. As an example, consider Fig. 10 where $\Omega=0.5$ and $n=8, l=3$ and $m=11$, satisfying $m=n+l$. It should be noted that the relation- 
ship $m=n+l$ still holds as the isolated coreless density peaks disappear from the density profile of component-2; this is because the phase imprints due to the vortices in component-1, which constitute $l$, are still present.

\section{A Non-Linear $\sigma$-Model}

The ground states of the rotating two-component condensate can be recovered from a 'non-linear $\sigma$-model' formulation of the energy functional in terms of the total density $\rho_{T}$ and spin vector $\mathbf{S}$ (Eq.'s (9)-(10)). We will analyze in detail the possible regimes in this section and show how a Thomas Fermi approximation can hold in the case $\Gamma_{12}>0$ or can be generalized and provide relevant information in the case $\Gamma_{12}<0$.

\section{A. Energy Functional Representation}

We write the energy functional of the two wave functions (Eq. (11), non-dimensionalised) as $E\left[\psi_{1}, \psi_{2}\right]=$ $E_{\mathrm{KE}}+E_{\mathrm{PE}}+E_{\mathrm{I}}$ where

$$
\begin{aligned}
E_{\mathrm{KE}}= & \frac{1}{\eta+1} \int\left|\left(\nabla-\frac{i}{2}(\eta+1) \boldsymbol{\Omega} \times \boldsymbol{r}\right) \psi_{1}\right|^{2} \\
& +\eta\left|\left(\nabla-\frac{i}{2 \eta}(\eta+1) \boldsymbol{\Omega} \times \boldsymbol{r}\right) \psi_{2}\right|^{2} d^{2} r \\
E_{\mathrm{PE}}= & \int 2 j_{1} r^{2}\left|\psi_{1}\right|^{2}+2 j_{2} r^{2}\left|\psi_{2}\right|^{2} \quad d^{2} r \\
E_{\mathrm{I}}= & \int \frac{1}{2} g_{1}\left|\psi_{1}\right|^{4}+\frac{1}{2} g_{2}\left|\psi_{2}\right|^{4}+g_{12}\left|\psi_{1}\right|^{2}\left|\psi_{2}\right|^{2} \quad d^{2} r
\end{aligned}
$$

with

$$
\begin{aligned}
& j_{1}=\frac{1}{2} \frac{(1+\eta) \xi^{2}}{(1+\xi)^{2}}-\frac{1}{8}(1+\eta) \Omega^{2}, \\
& j_{2}=\frac{1}{2} \frac{(1+\eta)}{\eta(1+\xi)^{2}}-\frac{1}{8 \eta}(1+\eta) \Omega^{2} .
\end{aligned}
$$

It is assumed that the $g_{k}, m_{k}, \omega_{k}$ and $N_{k}$ are distinct so that a weighted total density can be defined as (9) with $\psi_{1}=\sqrt{\rho_{T}} \chi_{1}$ and $\psi_{2}=\sqrt{\rho_{T} / \eta} \chi_{2}$. The spin density vector $\boldsymbol{S}$, which satisfies $|\boldsymbol{S}|^{2}=1$ everywhere, has components given by Eq.'s (10). We then have

$$
\left|\psi_{1}\right|^{2}=\frac{1}{2} \rho_{T}\left(1+S_{z}\right), \quad\left|\psi_{2}\right|^{2}=\frac{1}{2 \eta} \rho_{T}\left(1-S_{z}\right) .
$$

We introduce the phases $\theta_{1}$ and $\theta_{2}$ defined by $\chi_{1}=$ $\left|\chi_{1}\right| \exp \left(i \theta_{1}\right)$ and $\chi_{2}=\left|\chi_{2}\right| \exp \left(i \theta_{2}\right)$. The energy functional is expressed in terms of 4 variables: the total density $\rho_{T}$, the component $S_{z}$ and the angles $\theta_{1}, \theta_{2}$. We see that $E_{\mathrm{KE}}=E_{\rho_{T}}+E_{\mathrm{Sz}}+E_{\theta_{1}, \theta_{2}}$ where

$$
\begin{aligned}
E_{\rho_{T}} & =\int \frac{1}{(\eta+1)}\left(\nabla \sqrt{\rho_{T}}\right)^{2} \quad d^{2} r, \\
E_{\mathrm{Sz}}= & \int \frac{1}{4} \frac{\rho_{T}}{(\eta+1)} \frac{\left(\nabla S_{z}\right)^{2}}{\left(1-S_{z}^{2}\right)} d^{2} r \\
E_{\theta_{1}, \theta_{2}}= & \int \frac{1}{2} \frac{\rho_{T}}{(1+\eta)}\left[\left(1+S_{z}\right)\left(\nabla \theta_{1}-\frac{1}{2}(1+\eta) \boldsymbol{\Omega} \times \boldsymbol{r}\right)^{2}\right. \\
& \left.+\left(1-S_{z}\right)\left(\nabla \theta_{2}-\frac{1}{2 \eta}(1+\eta) \boldsymbol{\Omega} \times \boldsymbol{r}\right)^{2}\right] d^{2} r .
\end{aligned}
$$

The other terms of the energy straightforwardly become

$$
\begin{aligned}
E_{\mathrm{PE}} & =\int\left[\left(j_{1}+j_{2} / \eta\right)+\left(j_{1}-j_{2} / \eta\right) S_{z}\right] r^{2} \rho_{T} \\
E_{\mathrm{I}} & =\int \frac{\rho_{T}^{2}}{2}\left(\bar{c}_{0}+\bar{c}_{1} S_{z}+\bar{c}_{2} S_{z}^{2}\right) \quad d^{2} r
\end{aligned}
$$

with

$$
\begin{aligned}
& \bar{c}_{0}=\frac{1}{4 \eta^{2}}\left(\eta^{2} g_{1}+g_{2}+2 \eta g_{12}\right), \\
& \bar{c}_{1}=\frac{1}{2 \eta^{2}}\left(\eta^{2} g_{1}-g_{2}\right), \\
& \bar{c}_{2}=\frac{1}{4 \eta^{2}}\left(\eta^{2} g_{1}+g_{2}-2 \eta g_{12}\right) .
\end{aligned}
$$

Thus the complete energy is

$$
\begin{aligned}
E & =\int \frac{1}{(\eta+1)}\left(\nabla \sqrt{\rho_{T}}\right)^{2}+\frac{\rho_{T}}{4(\eta+1)} \frac{\left(\nabla S_{z}\right)^{2}}{\left(1-S_{z}^{2}\right)} \\
& +\frac{\rho_{T}}{2(1+\eta)} \times \\
& {\left[\left(1+S_{z}\right)\left(\nabla \theta_{1}-\frac{1}{2}(1+\eta) \boldsymbol{\Omega} \times \boldsymbol{r}\right)^{2}\right.} \\
& \left.+\left(1-S_{z}\right)\left(\nabla \theta_{2}-\frac{1}{2 \eta}(1+\eta) \boldsymbol{\Omega} \times \boldsymbol{r}\right)^{2}\right] \\
& +\left[\left(j_{1}+j_{2} / \eta\right)+\left(j_{1}-j_{2} / \eta\right) S_{z}\right] r^{2} \rho_{T} \\
& +\frac{\rho_{T}^{2}}{2}\left(\bar{c}_{0}+\bar{c}_{1} S_{z}+\bar{c}_{2} S_{z}^{2}\right) \quad d^{2} r .
\end{aligned}
$$

Energy (17) is subject to the constraints (4) that can be rewritten as

$$
\begin{array}{ll}
\int \rho_{T}\left(1-S_{z}\right) / 2 & d^{2} r=N_{2} \eta \\
\int \rho_{T}\left(1+S_{z}\right) / 2 & d^{2} r=N_{1} .
\end{array}
$$


or equivalently

$$
\begin{aligned}
\int \rho_{T} & d^{2} r=N_{1}+N_{2} \eta, \\
\int \rho_{T} S_{z} & d^{2} r=N_{1}-N_{2} \eta
\end{aligned}
$$

The minimization of the energy under the constraints (19) yields two coupled equations with two Lagrange multipliers, $\mu$ and $\lambda$. We will write them when the phase is constant (the gradient of the phases $\theta_{1}$ and $\theta_{2}$ can be ignored) and the effect of rotation is contained in an effective centrifugal dilation of the trapping potential included in $j_{1}, j_{2}$ :

$$
\begin{aligned}
\mu+\lambda S_{z}=- & \frac{1}{(1+\eta)} \frac{\Delta \sqrt{\rho_{T}}}{\sqrt{\rho_{T}}}+\frac{1}{4(1+\eta)} \frac{\left(\nabla S_{z}\right)^{2}}{\left(1-S_{z}^{2}\right)} \\
+ & {\left[\left(j_{1}+j_{2} / \eta\right)+\left(j_{1}-j_{2} / \eta\right) S_{z}\right] r^{2} } \\
& +\rho_{T}\left(\bar{c}_{0}+\bar{c}_{1} S_{z}+\bar{c}_{2} S_{z}^{2}\right),
\end{aligned}
$$

and

$$
\begin{aligned}
\lambda=- & \frac{1}{4(1+\eta)} \frac{\Delta S_{z}+\nabla \rho_{T} \cdot \nabla S_{z}}{\left(1-S_{z}^{2}\right)}+\frac{1}{2(1+\eta)} \frac{S_{z}\left(\nabla S_{z}\right)^{2}}{\left(1-S_{z}^{2}\right)} \\
& +\left(j_{1}-j_{2} / \eta\right) r^{2}+\left(\bar{c}_{1}+2 \bar{c}_{2} S_{z}\right) \rho_{T} / 2 .
\end{aligned}
$$

As pointed out in [14], in the general case where $\eta$ and $\xi$ are different from 1, we have seen from the expression $E_{\theta_{1}, \theta_{2}}$ that the effective velocity in each component is different. Nevertheless, in the case when $\eta=\xi=1$, it is possible to define an effective velocity shared by both components,

$$
v_{\text {eff }}=\frac{\nabla \Theta}{2}+\frac{\boldsymbol{R} S_{z}}{2\left(1-S_{z}^{2}\right)}
$$

where $\Theta=\theta_{1}+\theta_{2}$ and $\boldsymbol{R}=S_{y} \nabla S_{x}-S_{x} \nabla S_{y}$. We note the identity

$$
\frac{\left(\nabla S_{z}\right)^{2}}{\left(1-S_{z}^{2}\right)}=(\nabla \boldsymbol{S})^{2}-\frac{R^{2}}{\left(1-S_{z}^{2}\right)},
$$

where $(\nabla \boldsymbol{S})^{2}=\left(\nabla S_{x}\right)^{2}+\left(\nabla S_{y}\right)^{2}+\left(\nabla S_{z}\right)^{2}$. Expansion of the square in $E_{\theta_{1}, \theta_{2}}$, substituting in the $v_{\text {eff }}$ and using the identity from above reduces the energy to the simple form found in [19, 24].

$$
\begin{aligned}
E=\int \frac{1}{2}\left(\nabla \sqrt{\rho_{T}}\right)^{2}+\frac{\rho_{T}}{8}(\nabla \boldsymbol{S})^{2} & \\
+ & \frac{\rho_{T}}{2}\left(v_{\mathrm{eff}}-\boldsymbol{\Omega} \times \boldsymbol{r}\right)^{2}+\frac{1}{2} r^{2}\left(1-\Omega^{2}\right) \rho_{T} \\
& +\frac{\rho_{T}^{2}}{2}\left(c_{0}+c_{1} S_{z}+c_{2} S_{z}^{2}\right) \quad d^{2} r
\end{aligned}
$$

where $c_{0}, c_{1}$ and $c_{2}$ are equal to $\bar{c}_{0}, \bar{c}_{1}$ and $\bar{c}_{2}$ with $\eta$ set equal to unity.

\section{B. A Thomas-Fermi approximation}

The typical Thomas-Fermi (TF) approximation to Eq.'s (201)-(21) is to assume that derivatives in $\rho_{T}$ and $S_{z}$ are negligible in front of the other terms. If we apply the $\mathrm{TF}$ approximation we then get

$$
\begin{gathered}
\mu+\lambda S_{z}=\left[\left(j_{1}+j_{2} / \eta\right)+\left(j_{1}-j_{2} / \eta\right) S_{z}\right] r^{2} \\
+\rho_{T}\left(\bar{c}_{0}+\bar{c}_{1} S_{z}+\bar{c}_{2} S_{z}^{2}\right),
\end{gathered}
$$

and

$$
\lambda=\left(j_{1}-j_{2} / \eta\right) r^{2}+\frac{1}{2}\left(\bar{c}_{1}+2 \bar{c}_{2} S_{z}\right) \rho_{T} .
$$

The TF energy is then

$$
\begin{aligned}
E_{\mathrm{TF}}=\int & {\left[\left(j_{1}+j_{2} / \eta\right)+\left(j_{1}-j_{2} / \eta\right) S_{z}\right] r^{2} \rho_{T} } \\
& +\frac{\rho_{T}^{2}}{2}\left(\bar{c}_{0}+\bar{c}_{1} S_{z}+\bar{c}_{2} S_{z}^{2}\right)-\mu \rho_{T}-\lambda \rho_{T} S_{z} \quad d^{2} r .
\end{aligned}
$$

It is important to point out that the reduction of this quadratic form in $\rho_{T}$ and $\rho_{T} S_{z}$ yields

$$
\begin{aligned}
E_{\mathrm{TF}}=\int \frac{\bar{c}_{2}}{2}\left(\rho_{T} S_{z}+\frac{\bar{c}_{1}}{2 \bar{c}_{2}} \rho_{T}+\frac{1}{\bar{c}_{2}}\left(j_{1}-j_{2} / \eta\right) r^{2}-\frac{\lambda}{\bar{c}_{2}}\right)^{2} \\
+\frac{1}{2}\left(\bar{c}_{0}-\frac{\bar{c}_{1}^{2}}{4 \bar{c}_{2}}\right) \times \\
\quad\left[\rho_{T}-\frac{1}{\left(\bar{c}_{0}-\bar{c}_{1}^{2} / 4 \bar{c}_{2}\right)}\left(\mu-\left(j_{1}+j_{2} / \eta\right) r^{2}\right)\right. \\
+ \\
\left.+\frac{1}{2} \frac{\bar{c}_{1}}{\left(\bar{c}_{0} \bar{c}_{2}-\bar{c}_{1}^{2} / 4\right)}\left(\lambda-\left(j_{1}+j_{2} / \eta\right) r^{2}\right)\right]^{2} d^{2} r \\
+ \\
+ \text { constant terms. }
\end{aligned}
$$

The existence of a minimum for this quadratic form implies that $\bar{c}_{2} \geq 0$ and $\bar{c}_{0}-\bar{c}_{1}^{2} /\left(4 \bar{c}_{2}\right) \geq 0$. Since $\bar{c}_{0}-\bar{c}_{1}^{2} /\left(4 \bar{c}_{2}\right)=g_{1} g_{2} \Gamma_{12} /\left(4 \eta^{2} \bar{c}_{2}\right)$, this implies in particular that $\Gamma_{12}>0$.

Therefore, if $\Gamma_{12}>0$, (which implies $\bar{c}_{2}>0$ ), a Thomas Fermi approximation can be performed as has previously been considered, generally taking an approximation on the individual component wave functions $\psi_{1}$ and $\psi_{2}$ [22, 36, 46 [51]. Nevertheless, as we will see below, if $\bar{c}_{2}<0$ and $\Gamma_{12}<0$, then $\left(\bar{c}_{0}-\frac{\bar{c}_{1}^{2}}{4 \bar{c}_{2}}\right)>0$ and a TF approximation can still be performed on $\rho_{T}$, provided we keep gradient terms in $S_{z}$.

Multiplying (26) by $S_{z}$ and subtracting (25) leads to

$$
\mu=\left(j_{1}+j_{2} / \eta\right) r^{2}+\rho_{T}\left(\frac{\bar{c}_{1}}{2} S_{z}+\bar{c}_{0}\right) .
$$

Simplification by $\rho_{T} S_{z}$ with (26) yields, when $\psi_{1} \times \psi_{2} \neq$ 
0 ,

$$
\rho_{T}=\frac{a_{3}+a_{4} r^{2}}{g_{1} g_{2} \Gamma_{12}}
$$

and

$$
S_{z}=\frac{a_{1}+a_{2} r^{2}}{a_{3}+a_{4} r^{2}}
$$

where

$$
\begin{aligned}
& a_{1}=4 \eta^{2}\left(\lambda \bar{c}_{0}-\mu \bar{c}_{1} / 2\right), \\
& a_{2}=\eta g_{1} h_{2}-g_{2} h_{1}, \\
& a_{3}=4 \eta^{2}\left(\mu \bar{c}_{2}-\lambda \bar{c}_{1} / 2\right), \\
& a_{4}=-\left(\eta g_{1} h_{2}+g_{2} h_{1}\right),
\end{aligned}
$$

and where we define

$$
h_{k}=2\left(j_{k}-\frac{g_{12}}{g_{3-k}} j_{3-k}\right) .
$$

When only one component is present $\left(\psi_{1} \times \psi_{2}=0\right)$, this simplifies to

$$
\rho_{T}= \begin{cases}\frac{1}{g_{1}}\left[\mu+\lambda-2 j_{1} r^{2}\right] & \text { if } \psi_{2}=0 \\ \frac{\eta}{g_{2}}\left[\eta(\mu-\lambda)-2 j_{2} r^{2}\right] & \text { if } \psi_{1}=0\end{cases}
$$

since $S_{z}=+1$ when $\psi_{2}=0$ and $S_{z}=-1$ when $\psi_{1}=0$.

To begin, we note from Eq. (31) that $r^{2}=\left(a_{1}-\right.$ $\left.a_{3} S_{z}\right) /\left(a_{4} S_{z}-a_{2}\right)$, so that

$$
\rho_{T}=\frac{a_{1} a_{4}-a_{2} a_{3}}{g_{1} g_{2} \Gamma_{12}\left(a_{4} S_{z}-a_{2}\right)} .
$$

Since the intracomponent coupling strengths are chosen to be distinct, the support of each component will not necessarily be equal. In order to lead the computations, we have to assume a geometry for the components: two disks, a disk and annulus or two half-balls (droplets) and a sign for $\Gamma_{12}$.

\section{C. $\Gamma_{12}>0$}

\section{Both components are disks}

We start when both components are disks. Without loss of generality, we can assume that the outer boundary of component-2 (at $r=R_{2}$ ) is larger than that of component-1 (at $r=R_{1}$ ). Therefore, $S_{z}=-1$ and (34) holds in the annulus, while (35) holds in the coexisting region, which is the inside disk. The integrals from Eq.'s
(18) then give

$$
\begin{aligned}
\frac{N_{1}}{2 \pi} & =\int_{0}^{R_{1}} \rho_{T} \frac{\left(1+S_{z}\right)}{2} \quad r d r \\
& =-\frac{\left(a_{1} a_{4}-a_{2} a_{3}\right)^{2}}{4 g_{1} g_{2} \Gamma_{12}} \int_{s_{0}}^{-1} \frac{\left(1+S_{z}\right)}{\left(a_{4} S_{z}-a_{2}\right)^{3}} \quad d S_{z},
\end{aligned}
$$

where $s_{0}=\left.S_{z}\right|_{r=0}=a_{1} / a_{3}$ and

$$
\begin{aligned}
\frac{\eta N_{2}}{2 \pi}= & \int_{0}^{R_{1}} \rho_{T} \frac{\left(1-S_{z}\right)}{2} \quad r d r+\int_{R_{1}}^{R_{2}} \rho_{T} \quad r d r \\
= & -\frac{\left(a_{1} a_{4}-a_{2} a_{3}\right)^{2}}{4 g_{1} g_{2} \Gamma_{12}} \int_{s_{0}}^{-1} \frac{\left(1-S_{z}\right)}{\left(a_{4} S_{z}-a_{2}\right)^{3}} \quad d S_{z} \\
& -\frac{g_{2}}{4 \eta j_{2}} \int_{\rho_{d}}^{0} \rho_{T} \quad d \rho_{T},
\end{aligned}
$$

with

$$
\begin{aligned}
\rho_{d} & =\left.\frac{a_{3}+a_{4} r^{2}}{g_{1} g_{2} \Gamma_{12}}\right|_{r=R_{1}} \\
& =\frac{a_{2} a_{3}-a_{1} a_{4}}{g_{1} g_{2} \Gamma_{12}\left(a_{2}+a_{4}\right)},
\end{aligned}
$$

since $S_{z}=-1$ at $r=R_{1}$.

Completion of these integrals and noting that $\left\{a_{2}+\right.$ $\left.a_{4}, a_{2}-a_{4}\right\}=\left\{-2 g_{2} h_{1}, 2 \eta g_{1} h_{2}\right\}$ gives

$$
a_{3}^{2}=\frac{8 N_{1} g_{1} g_{2}^{2} \Gamma_{12} h_{1}}{\pi\left(s_{0}+1\right)^{2}}
$$

which must necessarily be positive, i.e. $h_{1} \Gamma_{12}>0$, and

$$
\begin{aligned}
N_{1}\left(a_{2}-s_{0} a_{4}\right)^{2}=2 \eta^{2} & N_{2} g_{1} g_{2} j_{2} h_{1} \Gamma_{12}\left(1+s_{0}\right)^{2} \\
+ & 2 N_{1}\left(1+s_{0}\right) \eta j_{2} g_{1} \Gamma_{12} \times \\
& {\left[\eta g_{1} h_{2}\left(1+s_{0}\right)+2 g_{2} h_{1}\left(s_{0}-1\right)\right] }
\end{aligned}
$$

where in Eq. (38b) the expression for $a_{3}$ from Eq. (38a) has been substituted. This equation can always be solved in terms of $s_{0}$ when $h_{1} \Gamma_{12}$ is positive since the discriminant is equal to $8 N_{1} \eta^{2} g_{2}^{2} g_{1} j_{2} h_{1} \Gamma_{12}\left(N_{1} g_{12}+N_{2} g_{2}\right)$. We find that

$$
1+s_{0}=\frac{2 N_{1} g_{2}}{N_{1} g_{2}-\eta N_{1} g_{12}+\sqrt{2 N_{1} \eta^{2} g_{1} j_{2} \Gamma_{12}\left(N_{1} g_{12}+N_{2} g_{2}\right) / h_{1}}} .
$$

A similar calculation can be completed if the outer boundary of component-2 is larger than that of component-1. This then gives that $h_{2} \Gamma_{12}>0$ (although expressions (38) change slightly).

If an annulus develops in component $3-k$, it means that $s_{0}=1\left(s_{0}=-1\right)$ for $k=1(k=2)$. Inputting this 
choice into Eq. (38b) gives

$$
\begin{aligned}
\bar{g}_{12} & =\frac{N_{k} g_{k} j_{3-k}}{2\left(N_{1} j_{1}+N_{2} j_{2}\right)} \\
& +\frac{1}{2}\left(\left[\frac{N_{k} g_{k} j_{3-k}}{N_{1} j_{1}+N_{2} j_{2}}\right]^{2}+\frac{4 N_{3-k} g_{1} g_{2} j_{3-k}}{N_{1} j_{1}+N_{2} j_{2}}\right)^{1 / 2}
\end{aligned}
$$

as the critical $g_{12}$ at which an annulus forms in component- $\{3-k\}$. In the case of Fig. 7, the curve (39) has been plotted in dashed lines and is close to the numerical curve. Notice that if $\xi^{2}=1$ (equal trapping frequencies for both components), then Eq. (39) becomes independent of $\Omega$, as in the phase diagrams of Fig. 6. where it yields $\Gamma_{12}=0.008$.

Let us point out that before the transition to the disk plus annulus takes place, there is a subregion of region 1 , where there are 2 disks, but in one component the wave function has a local minimum at the origin. For instance, in the case of Fig. 6, it corresponds to $\Gamma_{1}$ changing sign.

As a conclusion, in order for the ground state to be composed of two disks, assuming that component- $k$ is the component with smaller support, we need that $h_{k}>0$, $\Gamma_{12}>0$ and $g_{12}<\bar{g}_{12}$. These three conditions can be summarised as

$$
g_{12}<\min \left(\frac{j_{k}}{j_{3-k}} g_{3-k}, \frac{j_{3-k}}{j_{k}} g_{k}, \bar{g}_{12}, \sqrt{g_{1} g_{2}}\right) \text { if } h_{1}, h_{2}>0,
$$

$\frac{j_{3-k}}{j_{k}} g_{k}<g_{12}<\min \left(\frac{j_{k}}{j_{3-k}} g_{3-k}, \bar{g}_{12}, \sqrt{g_{1} g_{2}}\right)$ if $h_{3-k}<0$.

\section{A disk and an annular component}

In this case, we can assume a disk in component-1 and an annulus in component-2; there are three regions: an inner disk where only component- 1 is present and (34) holds, an outer annulus where only component-2 is present and (34) holds and an inner annulus where both components coexist and (35) holds.

In order to use the TF approximation when one component is annular, computations similar to (38a)-(38b) lead to $h_{1} h_{2}<0$ and $g_{12}>\bar{g}_{12}$. This can be summarized (for an annulus in component- $\{3-k\}$ ) as

$$
\max \left(\frac{j_{3-k}}{j_{k}} g_{k}, \bar{g}_{12}\right)<g_{12}<\min \left(\frac{j_{k}}{j_{3-k}} g_{3-k}, \sqrt{g_{1} g_{2}}\right),
$$

with $h_{3-k}<0$. Equation (41) also places the restriction that

$$
\begin{aligned}
g_{k} & <g_{3-k}\left(\frac{j_{k}}{j_{3-k}}\right)^{2} \\
& =g_{3-k} \Lambda_{k},
\end{aligned}
$$

where $\Lambda_{k}=\left(j_{k} / j_{3-k}\right)^{2}$. Note that $\Lambda_{1} \Lambda_{2}=1$ such that an annulus develops in component-2 $(-1)$ if $g_{2}>(<) g_{1} \Lambda_{2}$.

\section{Orders of Intracomponent Strengths and Special Cases}

The effect that changing the order of the intracomponent strengths and particle numbers has on $\bar{g}_{12}$, and thus on the phase diagrams, is now investigated. There are two cases to consider, depending on the relative orders of the particle numbers. Drawing aid from experimental values, it is always expected that $\min \left\{N_{1}, N_{2}\right\} \gg$ $\max \left\{g_{1}, g_{2}\right\}$. Throughout it will be assumed that $g_{2}>$ $\Lambda_{2} g_{1}$, so the annulus develops in component-2 and that $j_{1}$ and $j_{2}$ are of order unity.

In the first case when the particle number of component- 1 is much greater than the particle number of component-2 $\left(N_{1} \gg N_{2}\right)$, it follows that $\bar{g}_{12} \sim g_{1} j_{2} / j_{1}$. The boundary between region 1 and region 3 is then directly dependent on the value of the ratio $j_{2} g_{1} /\left[j_{1} g_{2}\right]$. Notice that if $g_{2} \gg g_{1}, \Gamma_{12}$ evaluated at $g_{12}=\bar{g}_{12}$ tends to unity and as such an annulus would always be present in component-2, whatever the value of $g_{12}$.

Conversely in the second case when the particle number of component-1 is much smaller than the particle number of component-2 $\left(N_{1} \ll N_{2}\right)$, it follows that $\bar{g}_{12} \sim \sqrt{g_{1} g_{2}}$ which implies that the annulus will only develop near $\Gamma_{12}=0$.

We can also look at some special cases - there are four that can be considered:

Case (i). $\Lambda_{k} g_{3-k}=g_{k}$. When $\Lambda_{2}$ (equivalently $\Lambda_{1}$ ) is such that this equality is made, the two components are both disks and no annulus develops.

Case (ii). $\eta=\xi=1$. Then $\Lambda_{k}=1$ and $R_{2} \gtrless R_{1} \Longleftrightarrow$ $N_{2} g_{2} \Gamma_{1} \gtrless N_{1} g_{1} \Gamma_{1}$ when there are two disks and the annulus develops in the component which has the larger interaction strength.

Case (iii). $\eta=\xi=1$ and $g_{1}=g_{2} \equiv g$. When the intracomponent coupling strengths are equal, $\bar{g}_{12}=g$ and there are always two disks with $R_{2} \gtrless R_{1} \Longleftrightarrow N_{2} \gtrless$ $N_{1}$.

Case (iv). $\eta=\xi=1, g_{1}=g_{2} \equiv g$ and $N_{1}=N_{2} \equiv N$. When the particle numbers and intracomponent coupling strengths are equal, $\bar{g}_{12}=g$ and there are always two disks with $R_{2}=R_{1}$. A detailed analysis of this case, explored numerically in a phase diagram for all $\Gamma_{12}$ and analytically in the TF limit, was considered by [20]. 


\section{Justification of the Thomas-Fermi approximation}

The gradient terms in (20)-21) can be neglected if $d_{h l}$, the characteristic length of variation of $\rho_{T}$ and $S_{z}$ is much smaller than $d_{c}$, the characteristic size of the condensates. We have that $1 / d_{h l}^{2}$ is of order of $\mu$ and $\lambda$, which are of order $\sqrt{N_{k} g_{k}}, \sqrt{N_{k} g_{3-k}}$. Hence $d_{h l}$ is bounded above by the maximum of $\left(N_{k} g_{k}\right)^{-1 / 4},\left(N_{k} g_{3-k}\right)^{-1 / 4}$. From the expression of $a_{3}$, the characteristic size of the condensate is of order the minimum of $\left(g_{k} N_{k} \Gamma_{12}\right)^{1 / 4}$. Therefore, the Thomas Fermi approximation holds if $N_{k} g_{k} \sqrt{\Gamma_{12}}$, $N_{k} g_{3-k} \sqrt{\Gamma_{12}}$ are large. This requires the usual Thomas Fermi criterion that $N_{k} g_{k}, N_{k} g_{3-k}$ are large, but breaks down if $\Gamma_{12}$ is too small.

For $\Gamma_{12}=0$, the equations lead to spatial separation: either the radii of the disks tend to 0 in the case of two disks or the outer radius of the inner disk tends to the inner radius of the annulus in the case of disk plus annulus so that $\psi_{1} \times \psi_{2}=0$ everywhere. Another analysis has to be carried out to understand the region of coexistence, which is of small size and has strong gradients.

\section{D. $\Gamma_{12}<0$, beyond the TF approximation}

For negative $\Gamma_{12}$, and if $N_{k} g_{k}$ are large, the Thomas Fermi approximation can be extended provided some model takes into account the small region where the condensates coexist.

Indeed, if we go back to (28), and have both $\bar{c}_{2}<0$ and $\Gamma_{12}<0$, then the coefficient in front of the second square is positive and the optimal situation is to have the square equal to 0 , which leads to the inverted parabola (30). On the other hand, the coefficient in front of the first square is negative, and the ground state involves derivatives in $S_{z}$ to compensate it. Under the assumption that the boundary layer where $S_{z}$ varies is small, we are going to derive a TF model with jump for $\rho_{T}$. We will analyze it for the different geometries (disk plus annulus, droplets and vortex sheets) and show that it provides information consistent with the numerics.

\section{Disk Plus Annulus}

Assuming that the boundary layer is present only at some $r=r_{s}$, then $S_{z}=+1$ in the region in which component-2 is zero $\left(r \leq r_{s}^{-}\right)$and $S_{z}=-1$ in the region in which component-1 is zero $\left(r \geq r_{s}^{+}\right)$. The transition from $S_{z}=+1$ to $S_{z}=-1$ is not smooth, therefore creating the jump in density. Therefore, we are lead to (a)

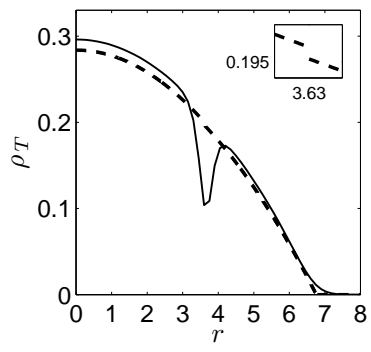

(b)

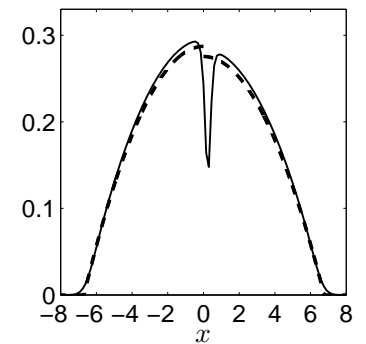

FIG. 11: Total density profiles (divided by $10^{4}$ ) obtained numerically (solid lines) and analytically (dashed lines) for two spatial separation cases with $g_{1}=0.0078, g_{2}=0.0083$, $N_{1}=N_{2}=10^{5}, \eta=\xi=1$ (set ES1) and $\Gamma_{12}=-10$ and $\Omega=0$ : (a) annulus plus disk, analytical estimate coming from (43) and (b) droplet, analytical estimate coming from (49). The inset in (a) shows the discontinuity of density at $r_{s}=3.63$. Distance is measured in units of $r_{0}$ and density in units of $r_{0}^{-2}$.

minimise the integral

$$
\begin{aligned}
I= & \int_{B_{r_{s}}} 2 j_{1} r^{2} \rho_{T}+\frac{g_{1}}{2} \rho_{T}^{2} \quad d^{2} r \\
& +\int_{B_{R \backslash r_{s}}} 2 \frac{j_{2}}{\eta} r^{2} \rho_{T}+\frac{g_{2}}{2 \eta^{2}} \rho_{T}^{2} \quad d^{2} r
\end{aligned}
$$

with respect to $r_{s}$. Here $B_{r_{s}}$ is a ball of radius $r_{s}$ and $B_{R \backslash r_{s}}$ is a torus with outer boundary at $r=R$ and inner boundary at $r=r_{s}$. Thus

$$
\begin{aligned}
2 \pi r_{s} & {\left.\left[2 j_{1} r^{2} \rho_{T}+\frac{g_{1}}{2} \rho_{T}^{2}\right]\right|_{r=r_{s}^{-}} } \\
& -\left.2 \pi r_{s}\left[2 \frac{j_{2}}{\eta} r^{2} \rho_{T}+\frac{g_{2}}{2 \eta^{2}} \rho_{T}^{2}\right]\right|_{r=r_{s}^{+}}=0,
\end{aligned}
$$

which implies that

$$
\begin{array}{ll}
\rho^{-}=\frac{1}{g_{1}}\left[\mu_{1}-2 j_{1} r^{2}\right] & r \in B_{r_{s}} \\
\rho^{+}=\frac{\eta}{g_{2}}\left[\mu_{2}-2 j_{2} r^{2}\right] & r \in B_{R \backslash r_{s}} .
\end{array}
$$

Then using the normalisation conditions $\int \rho^{-} d^{2} r=N_{1}$ and $\int \rho^{+} d^{2} r=\eta N_{2}$ we get an outer radius

$$
R=\left(r_{s}^{2}+\sqrt{\frac{g_{2} N_{2}}{\pi j_{2}}}\right)^{1 / 2}
$$


and chemical potentials

$$
\begin{aligned}
& \mu_{1}=\frac{N_{1} g_{1}}{\pi r_{s}^{2}}+j_{1} r_{s}^{2}, \\
& \mu_{2}=2 j_{2}\left(r_{s}^{2}+\sqrt{\frac{g_{2} N_{2}}{\pi j_{2}}}\right) .
\end{aligned}
$$

It remains to find $r_{s}$. But, from (44), it follows that

$$
r_{s}^{2}=\frac{\frac{4 N_{2} j_{2}}{\pi}-g_{1}\left(\frac{N_{1}}{\pi r_{s}^{2}}-\frac{j_{1} r_{s}^{2}}{g_{1}}\right)^{2}}{4\left[j_{1}\left(\frac{N_{1}}{\pi r_{s}^{2}}-\frac{j_{1} r_{s}^{2}}{g_{1}}\right)-2 j_{2} \sqrt{\frac{N_{2} j_{2}}{\pi g_{2}}}\right]} .
$$

The above leaves a quartic equation in $r_{s}^{2}$, the solution of which can be found numerically.

We can then complete the energy calculation to give

$$
I=\frac{g_{1} N_{1}^{2}}{2 \pi r_{s}^{2}}+\left(N_{1} j_{1}+2 N_{2} j_{2}\right) r_{s}^{2}-\frac{\pi j_{1}^{2}}{6 g_{1}} r_{s}^{6}+\frac{4}{3}\left(\frac{N_{2}^{3} g_{2} j_{2}}{\pi}\right)^{1 / 2} .
$$

A check plot of the density profile with the parameters $g_{1}=0.0078, g_{2}=0.0083$ and $N_{1}=N_{2}=10^{5}$ where $\eta=$ $\xi=1$ agrees well with this model where the boundary is calculated to be at $r_{s}=3.62$ (see Fig [1](a)).

\section{Droplets}

This formalism can also be extended to study the droplet case. As before, assuming a thin boundary layer, one can allow for a jump in density. We thus need to minimise the Thomas-Fermi energy for two droplets described by $B_{1}$ and $B_{2}$ that exist in the regions $0 \leq \theta \leq \alpha$ and $\alpha \leq \theta \leq 2 \pi$ respectively. The energy is then

$$
\begin{aligned}
I= & \alpha \int_{0}^{R_{1}} 2 j_{1} r^{2} \rho_{T}+\frac{g_{1}}{2} \rho_{T}^{2} \quad r d r \\
& +(2 \pi-\alpha) \int_{0}^{R_{2}} 2 \frac{j_{2}}{\eta} r^{2} \rho_{T}+\frac{g_{2}}{2 \eta^{2}} \rho_{T}^{2} \quad r d r .
\end{aligned}
$$

The expressions for the density in each domain (45) and the normalisation conditions allow completion of this integral to give

$$
I=\frac{4 \sqrt{2}}{3}\left[\left(\frac{N_{1}^{3} g_{1} j_{1}}{\alpha}\right)^{1 / 2}+\left(\frac{N_{2}^{3} g_{2} j_{2}}{(2 \pi-\alpha)}\right)^{1 / 2}\right] .
$$

It remains to find the optimum $\alpha$. This is achieved through the condition $d I / d \alpha=0$ which gives

$$
\begin{aligned}
\frac{\left(N_{1}^{3} g_{1} j_{1}\right)^{1 / 2}}{\alpha^{3 / 2}} & =\frac{\left(N_{2}^{3} g_{2} j_{2}\right)^{1 / 2}}{(2 \pi-\alpha)^{3 / 2}} \\
\Rightarrow \alpha & =2 \pi \frac{\bar{N}(\bar{g} \bar{j})^{1 / 3}}{\left(1+\bar{N}(\bar{g} \bar{j})^{1 / 3}\right)}
\end{aligned}
$$

where we have set $\bar{N}=N_{1} / N_{2}, \bar{g}=g_{1} / g_{2}$ and $\bar{j}=$ $j_{1} / j_{2}$. As expected, equality of the $N_{k}, g_{k}$ and setting $\eta=\xi=1\left(\Rightarrow j_{1}=j_{2}\right)$ gives $\alpha=\pi$ and the condensate is then composed of two half-balls. Otherwise, a curvature is present. A check plot of the density profile with the parameters $g_{1}=0.0078, g_{2}=0.0083$ and $N_{1}=N_{2}=10^{5}$ where $\eta=\xi=1$ is given in Fig 11(b).

Finally we can note the energy for the droplets is

$$
\begin{aligned}
I= & \frac{4}{3 \sqrt{\pi}}\left(1+\bar{N}(\bar{g} \bar{j})^{1 / 3}\right)^{-1 / 2} \times \\
& {\left[\left(N_{1}^{3} g_{1} j_{1}\right)^{1 / 2}+\left(N_{1} N_{2}^{2}\left(g_{1} g_{2}^{2} j_{1} j_{2}^{2}\right)^{1 / 3}\right)^{1 / 2}\right] . }
\end{aligned}
$$

This energy can be compared to the energy of the disk plus annulus (48) to determine which is the optimum geometry. Indeed, in the numerical cases studied before, the droplets are preferred states for small $\Omega$.

\section{Regions of Vortex Sheets}

In the case of vortex sheets, we can assume that the global profile of the total density is TF-like, obeying Eq. (30) in the bulk of the condensate. By working with the total density, we do not require any information on the vortex sheets themselves (and consequently $S_{z}$ ).

We thus take the form of $\rho_{T}$ from Eq. (30) from which we note that the outer boundary at $r=R$ satisfies $R=\sqrt{-a_{3} / a_{4}}$ and that completion of the normalisation condition (19a) gives

$$
a_{3}=\sqrt{-\frac{2\left(N_{1}+\eta N_{2}\right) g_{1} g_{2} \Gamma_{12} a_{4}}{\pi}} .
$$

This expression evidently requires $a_{4} \Gamma_{12}<0$. We however expect the vortex sheets to be present only in the $\Gamma_{12}<0$ domain, thus we can be more specific on the condition, namely that $a_{4}>0$, or

$$
g_{12}>\frac{\eta g_{1} j_{2}+g_{2} j_{1}}{\left(\eta j_{1}+j_{2}\right)} .
$$

We point out that this critical number corresponds to $\Gamma_{12}=0$ in the cases studied above. With $a_{3}$ as above and $a_{4}$ given by the parameters of the system, the density profile is then fully accessible.

\section{E. Analysis of defects}

The advantage of the Thomas Fermi analysis of $\rho_{T}$ in the nonlinear sigma model is that it allows for analysis of defects as a perturbation calculation when $N_{k} g_{k}, N_{k} g_{3-k}$ and $N_{k} g_{12}$ are large, in the spirit of what has been done for a single condensate in [44, 52 54] or for two condensates in [45]. We do not need to analyze specifically the 
peaks because they are taken into account in the $S_{z}$ formulation. This has been attacked in a more complicated fashion in the case of a single coreless vortex in 19]. We will develop our ideas in a later work but let us point out that the starting point is the energy (17) and if we call $p_{i}$ and $q_{i}$ the location of the vortex in each component, the main terms coming from the vortex contributions lead to:

- Each vortex core of each component providing a kinetic energy term proportional to $\rho_{T}(0) \log d_{h l} /(2(1+\eta))$ where $d_{h l}$ is the healing length, the characteristic size of the vortex core, here of order $1 / \sqrt{N_{k} g_{k}}$.

- the rotational term providing a term in $-c \Omega\left(\rho_{T}(0)\right)^{2}$, where $c$ is a numerical constant. The balance of these two terms allows us to compute the critical velocity for the nucleation of the first vortex.

- the kinetic energy then yielding a term in $-\log \mid p_{i}-$ $p_{j}|-\log | q_{i}-q_{j} \mid$.

- the rotation term yielding a term in $\Omega\left(\left|p_{i}\right|^{2}+\left|q_{i}\right|^{2}\right)$.

- the interaction term providing a term $\rho_{T}^{2} S_{z}^{2}$ for the perturbation of $S_{z}$ close to a coreless vortex. The ansatz can be made in several ways leading to an interaction term in $e^{-\left|p_{i}-q_{i}\right|^{2}}$.

This leads to a point energy of the type

$\sum_{i} a\left(\left|p_{i}\right|^{2}+\left|q_{i}\right|^{2}+b e^{-\left|p_{i}-q_{i}\right|^{2}}\right)-\sum_{i, j}\left(\log \left|p_{i}-p_{j}\right|+\log \left|q_{i}-q_{j}\right|\right)$

where $a$ and $b$ are related to the parameters of the problem. The ground state of such a point energy leads to a square lattice for a sufficient number of points and for some range of $a$ and $b$.

\section{Phase Diagram Under Conserved Total Particle Number}

In the experiment of Hall et al 2] a single component BEC of ${ }^{87} \mathrm{Rb}$ in the $|1,-1\rangle$ state was initially created, before a transfer of any desired fraction of the atoms from this $|1,-1\rangle$ state to the $|2,1\rangle$ state created the two-component BEC. Thus the ratio of particle numbers $N_{1} / N_{2}$ is controllable experimentally, with the constraint that $N_{1}+N_{2}$ is constant (in the case of the experiment of [2], $N_{1}+N_{2}=5 \times 10^{5}$ ). As such, experimentally, it is possible to keep the individual particle numbers constant (as in the normalisation condition (44)) or to keep the total particle number $N_{1}+N_{2}$ constant, allowing $N_{1}$ and $N_{2}$ to vary (as in (5)). We produce an $\Omega-\Gamma_{12}$ phase diagram for the parameters $g_{1}=0.003, g_{2}=0.006$ and $\eta=\xi=1$ using the normalisation condition given by
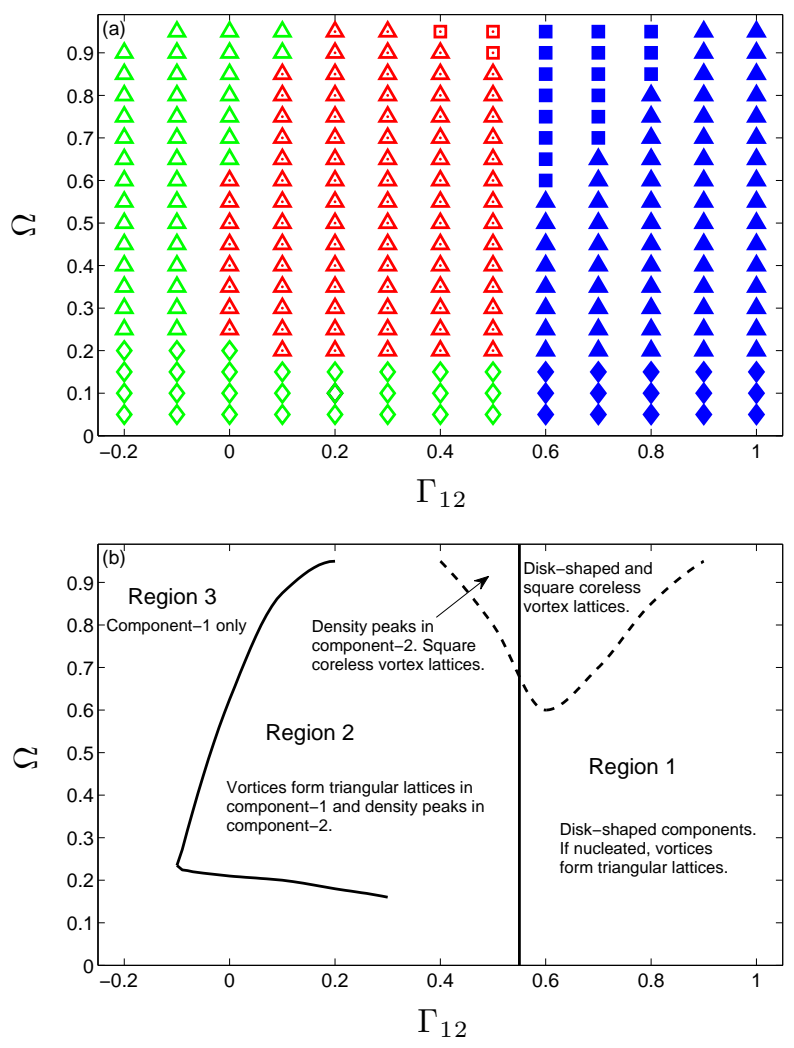

FIG. 12: (Color online) $\Omega-\Gamma_{12}$ phase diagram for parameters $g_{1}=0.003$ and $g_{2}=0.006$ with $\eta=\xi=1$ using the normalisation condition given by (5). (a) Numerical simulations where triangles indicate that the lattice in both components is triangular, squares that the lattice in both components is square and diamonds where no vortices have been nucleated. Filled triangles, squares and diamonds are where the two components are disk-shaped and coexist, empty triangles, squares and diamonds are where only component-1 exists; those with a dot in the centre represent the appearance of coreless vortices in component- 2 and those without a dot in the centre represent the complete disappearance of component-2. (b) A schematic representation of the numerical simulations. The solid lines indicate the boundary between different identified regions (determined by the geometry of the ground state) and the dashed lines the boundary between triangular and square lattices. The unit of rotation is $\tilde{\omega}$.

(5) with $N_{1}+N_{2}=2.1 \times 10^{5}$. Note that if $g_{1}=g_{2}$, $m_{1}=m_{2}$ and $\omega_{1}=\omega_{2}(\eta=\xi=1)$, then the phase diagram would be identical to that of Fig. 8 (i.e. the normalisation condition in this case would not be important). The $\Omega-\Gamma_{12}$ phase diagram is presented in Fig. 12 There are three distinct regions (determined by the geometry of the ground state):

Region 1. In the first region, both components are diskshaped. As before, the coreless vortices can either form a triangular or a square lattice depending on the values of $\Gamma_{12}$ and $\Omega$. Figure 13 shows this case for $\Gamma_{12}=0.7$ and $\Omega=0.65$ (where a triangular lattice is present) and 

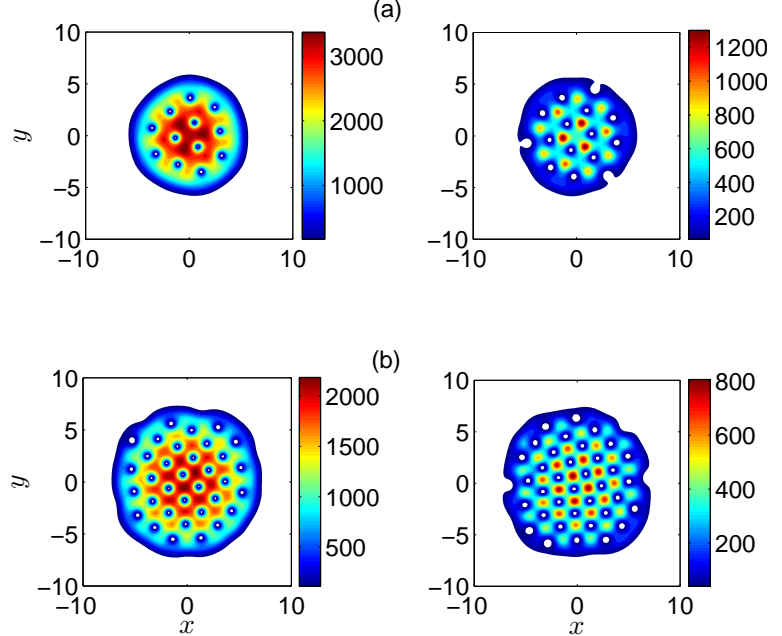

(b)

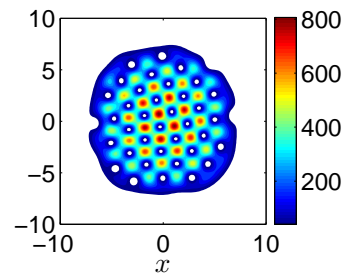

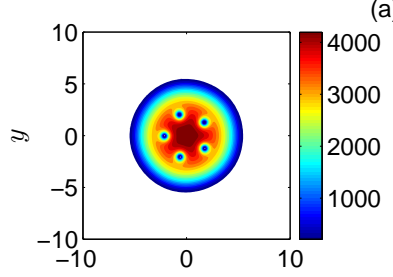
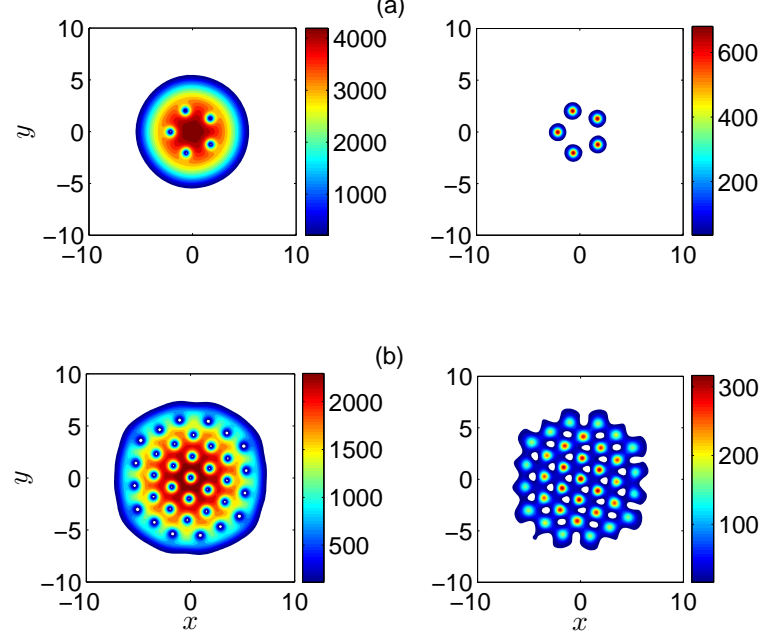

(b)

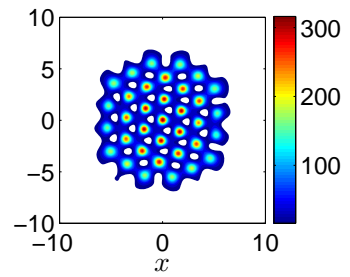

FIG. 13: (Color online) A series of density plots for component-1 (left column) and component-2 (right column). The parameters are $g_{1}=0.003$ and $g_{2}=0.006$ with $\eta=\xi=1$ and $\Gamma_{12}=0.7$ (which gives $g_{12}=0.0023$ ) and normalisation taken over the total density (Eq. (5)). The angular velocity of rotation is $\Omega$ and it takes the values (a) 0.65 and (b) 0.9 . There is a triangular lattice in (a) and a square lattice in (b). At these parameters the components are in region 1 of the phase diagram Fig. 12. Distance is measured in units of $r_{0}$ and density in units of $r_{0}^{-2}$.

$\Omega=0.9$ (where a square lattice is present). In region 1 , both components have the same radii.

Region 2. In the second region, only component1 exists except for isolated density peaks that exist in component-2. These isolated density peaks occur at the same location as the vortices do in component-1, and are thus identical to the isolated coreless vortices described in detail in Sect. IV. Figure 14 shows this case $\Gamma_{12}=0.3$ with $\Omega=0.5$ and $\Omega=0.9$.

Region 3. In the third region, only component-1 exists (the density peaks in component-2 that were present in region 2 are no longer present). Furthermore, only triangular vortex lattices are observed in this effective one component condensate (in component-1).

Computations similar to Section III hold, except that now we have to set $\lambda=0$. This leads to $a_{1} / a_{3}=$ $-\bar{c}_{1} /\left(2 \bar{c}_{2}\right)$. We see that this ratio (which is $\left.S_{z}(0)\right)$, reaches 1 or -1 when $\bar{c}_{1}= \pm 2 \bar{c}_{2}$. In our numerical case, this leads to $\bar{\Gamma}_{12}=0.5$. We see clearly 3 regimes: $\Gamma_{12}>\bar{\Gamma}_{12}$, where the condensates are 2 disks, $\Gamma_{12}<0$, which is phase separation, in which case $S_{z}=1$ is the preferred state and $0<\Gamma_{12}<\bar{\Gamma}_{12}$, in which case the TF approximation leads to a computation of $\rho_{T}$ with coreless vortex lattices and variations in $S_{z}$ which improve the energy and lead to this intermediate state, still to be
FIG. 14: (Color online) A series of density plots for component-1 (left column) and component-2 (right column). The parameters are $g_{1}=0.003$ and $g_{2}=0.006$ with $\eta=\xi=1$ and $\Gamma_{12}=0.3$ (which gives $g_{12}=0.0035$ ) and normalisation taken over the total density (Eq. (5)). The angular velocity of rotation is $\Omega$ and it takes the values (a) 0.5 and (b) 0.9 . At these parameters the components are in region 2 of the phase diagram Fig. 12. Distance is measured in units of $r_{0}$ and density in units of $r_{0}^{-2}$.

studied in more detail.

\section{Conclusion}

We have presented phase diagrams of rotating two component condensates in terms of the angular velocity $\Omega$ and a nondimensionnalized parameter related to the coupling strengths $\Gamma_{12}=1-g_{12}^{2} /\left(g_{1} g_{2}\right)$. We have analyzed the various ground states and topological defects and have found four sets characterized by the symmetry preserving/symmetry breaking, coexistence or spatial separation of the components. When the geometry of the ground states is either two disks (coexistence of components, region 1) or a disk and an annulus (spatial separation keeping some symmetry, region 3), the topological defects are coreless vortex lattices (with possible stabilization of the square lattice) or giant skyrmions at the boundary interface between the two components. In the complete symmetry breaking case, we have found vortex sheets and droplets. The difference of masses or coupling strengths between the components can induce very different patterns.

We have introduced an energy (17) related to the total density and a pseudo spin vector. The minimization in a generalized Thomas Fermi approximation provides a lot 
of information on the ground states for general masses, trapping frequencies and coupling strengths. Some parts of the phase diagrams can be justified rigorously, both in the case $\Gamma_{12}>0$, which had been studied before, but also in the case $\Gamma_{12}<0$ with generalized models. This formulation of the energy should bring in the future more information on the defects.

\section{Acknowledgments}

The authors wish to thank Thierry Jolicoeur for useful discussions that took place for the duration of this work. They are very grateful to the referee for his careful reading of the manuscript and his appropriate comments. We acknowledge support from the French ministry Grant ANR-BLAN-0238, VoLQuan.

[1] Matthews, M. R. et al 1999 Phys. Rev. Lett. 83, 2498.

[2] Hall, D. S. et al 1998 Phys. Rev. Lett. 81, 1539.

[3] Anderson et al 2009 Phys. Rev. A. 80, 023603.

[4] Myatt, C. J. et al 1996 Phys. Rev. Lett. 78, 586.

[5] Delannoy, G. et al 2001 Phys. Rev. A 63, 051602.

[6] Maddaloni, P. et al 2000 Phys. Rev. Lett. 85, 2413.

[7] Schweikhard, V et al 2004 Phys. Rev. Lett. 93, 210403.

[8] Papp, S. B. et al 2008 Phys. Rev. Lett. 101, 040402.

[9] Modugno, G. et al 2002 Phys. Rev. Lett. 89, 190404.

[10] Thalhammer, G. et al 2008 Phys. Rev. Lett. 100, 210402.

[11] Ferrari, G. et al 2002 Phys. Rev. Lett. 89, 053202.

[12] Savage, C. M. and Ruostekoski, J. 2003 Phys. Rev. Lett. 91, 010403.

[13] Ruostekoski, J. and Anglin, J. R. 2001 Phys. Rev. Lett. 86, 3934.

[14] Cooper, N. R. 2008 Advances in Physics 57, 539-616.

[15] Williams, J. E. and Holland, M. J. 1999 Nature 401, 568.

[16] Mertes, K. M. et al 2007 Phys. Rev. Lett. 99, 190402.

[17] Manton, N. and Sutcliffe, P. 2004. Topological Solitons, Cambridge University Press.

[18] Cho, Y. M. et al 2005 Phys. Rev. A 72, 063603.

[19] Kasamatsu, K., Tsubota, M. and Ueda, M. 2005 Int. J. Mod. Phys. B 19, 1835.

[20] Kasamatsu, K., Tsubota, M. and Ueda, M. 2003 Phys. Rev. Lett. 91, 150406.

[21] Yang, S., Wu, Q., Zhang, S. and Feng, S. 2008 Phys. Rev. A 77, 033621.

[22] Kasamatsu, K., Yasui, Y. and Tsubota, M. 2001 Phys. Rev. A 64, 053605.

[23] Ueda, M. and Kawaguchi, Y. 2010 arXiv, 1001.2072v2.
[24] Kasamatsu, K., Tsubota, M. and Ueda, M. 2005 Phys. Rev. A 71, 043611.

[25] Pitaevskii, L. and Stringari, S. 2003. International series of monographs in physics, 116, Oxford Science Publications.

[26] Tojo, S. et al 2010 Phys. Rev. A 82, 033609.

[27] Theis, M. et al 2004 Phys. Rev. Lett. 93, 123001.

[28] Bauer, D. M. et al 2009 Nature Physics 5, 339.

[29] van Kempen, E. G. M. et al 2002 Phys. Rev. Lett. 88, 093201.

[30] Kasamatsu, K. and Tsubota, M. 2009 Phys. Rev. A 79, 023606.

[31] Woo, S. J., Choi, S., Baksmaty, L. O. and Bigelow, N. P. 2007 Phys. Rev. A 75, 031604(R).

[32] Mueller, E. J. and Ho, T-L. 2002 Phys. Rev. Lett. 88, 180403.

[33] Keçeli, M. and Oktel, Ö. 2006 Phys. Rev. A 73, 023611.

[34] Barnett, R. et al 2008, New J. Phys. 10, 043030.

[35] Barnett, R. et al 2010, New J. Phys. 12, 043004.

[36] Ohberg, P. and Stenholm, S. 1997 Phys. Rev. A 57, 1272.

[37] Navarro et al 2009 Phys. Rev. A 80, 023613.

[38] Christensson, J. et al 2008, New J. Phys. 10, 033029.

[39] Ohberg, P. 1998 Phys. Rev. A 59, 634.

[40] For the parameters reported here, any vortices nucleated in the annulus of component-2 do not have corresponding density peaks outside of the circular component-1. However it is possible, using a different set of parameters, to create such density peaks outside of component-1.

[41] Eto, M., Kasamatsu, K., Nitta M., Takeuchi, H., Tsubota, M., 2011 Phys. Rev. A. 83, 063603.

[42] Catelani, G. and Yuzbashyan, E. A. 2010 Phys. Rev. A 81, 033629.

[43] Ohmi, T. and Machida, K. 1998 J. Phys. Soc. Jpn. 67, 1822-1825.

[44] Lin, F. H. et al 2009 Acta Mathematica Scientia 29B(3), 751-776.

[45] Lin, T. C. and Wei, J. 2007 Journal of Mathematical Physics 48, no.5, 053518.

[46] Trippenbach, M. et al 2000 J. Phys. B: At. Mol. Opt. Phys. 33, 4017.

[47] Corro, I., Scott, R. G. and Matrin, A. M. 2009 Phys. Rev. A 80, 033609.

[48] Ho, T-L. and Shenoy, V. B. 1996 Phys. Rev. Lett. 77, 3276.

[49] Pu, H. and Bigelow, N. P. 1998 Phys. Rev. Lett. 80, 1130.

[50] Riboli, F. and Modugno, M. 2002 Phys. Rev. A 65, 063614.

[51] Jezek, D. M. and Capuzzi, P. 2002 Phys. Rev. A 66, 015602.

[52] Aftalion, A. and Riviere, T. 2001 Phys. Rev. A 64, 043611.

[53] Aftalion, A. and Du, Q. 2001 Phys. Rev. A 64, 063603.

[54] Castin, Y. and Dum, R. 1998 Phys. Rev. A 57, 3008. 